\title{
Towards representation stability for the second homology of the Torelli group
}

\author{
SøREN K BOLDSEN \\ Mia Hauge Dollerup
}

\begin{abstract}
We show for $g \geq 7$ that the second homology group of the Torelli group, $H_{2}\left(\mathcal{I}_{g, 1} ; \mathbb{Q}\right)$, is generated as an $\mathrm{Sp}(2 g, \mathbb{Z})$-module by the image of $H_{2}\left(\mathcal{I}_{6,1} ; \mathbb{Q}\right)$ under the stabilization map. In the process we also show that the quotient $B\left(F_{g, i} ; i\right) / \mathcal{I}_{g, i}$ by the Torelli group of the complex of arcs with identity permutation is $(g-2)$-connected for $i=1,2$.
\end{abstract}

20C12, 20J06

\section{Introduction}

Let $F_{g, r}$ denote a smooth compact connected oriented surface of genus $g$ and $r$ boundary components. Let $\Gamma_{g, r}=\Gamma\left(F_{g, r}\right)$ denote its mapping class group, ie $\Gamma(F)=\pi_{0}\left(\operatorname{Diff}^{+}(F, \partial F)\right)$, where $\operatorname{Diff}^{+}(F, \partial F)$ is the group of orientation-preserving diffeomorphisms of $F$ that restrict to the identity on $\partial F$.

The Torelli group $\mathcal{I}_{g, 1}$ is the subgroup of $\Gamma_{g, 1}$ defined by the exact sequence

$$
1 \longrightarrow \mathcal{I}_{g, 1} \longrightarrow \Gamma_{g, 1} \longrightarrow \operatorname{Sp}(2 g, \mathbb{Z}) \longrightarrow 1 \text {. }
$$

To define the Torelli group of a surface with more than one boundary component, we proceed as in Putman [7]. Suppose we have an embedding $S \longrightarrow F_{g, 1}$ such that $F_{g, 1} \backslash S$ is connected. Write $\Gamma\left(F_{g, 1}, S\right)$ for the image of $\Gamma(S)$ in $\Gamma_{g, 1}$ under the map induced by this embedding. Then one defines

$$
\mathcal{I}\left(F_{g, 1}, S\right):=\mathcal{I}\left(F_{g, 1}\right) \cap \Gamma\left(F_{g, 1}, S\right) .
$$

Here we are interested in the case $S=F_{g-1,2}$, and the embedding is $\Sigma_{1,-1}: F_{g-1,2} \longrightarrow$ $F_{g, 1}$ which glues on a pair of pants. We write $\mathcal{I}_{g-1,2}$ for $\mathcal{I}\left(F_{g, 1}, F_{g-1,2}\right)$ defined via this embedding.

There is an exact sequence similar to (1), as follows: Let $\beta$ an arc such that $F_{g-1,2} \longrightarrow$ $F_{g, 1}$ is the inclusion of the cut-up surface $\left(F_{g, 1}\right)_{\beta} \longrightarrow F_{g, 1}$ as on Figure 1, denote 
by $\tilde{\beta}$ the closing-up of $\beta$ (see Figure 4 ), and let $b=[\widetilde{\beta}] \in H_{1}\left(F_{g, 1} ; \mathbb{Z}\right.$ ) be its homology class. Then

$$
1 \longrightarrow \mathcal{I}_{g-1,2} \longrightarrow \Gamma\left(F_{g, 1}, F_{g-1,2}\right) \longrightarrow \mathrm{Sp}(2 g, \mathbb{Z})_{b} \longrightarrow 1,
$$

where $\operatorname{Sp}(2 g, \mathbb{Z})_{b} \subseteq \operatorname{Sp}(2 g, \mathbb{Z})$ is the stabilizer subgroup for $b$.

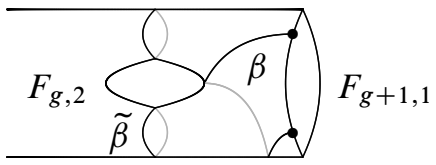

Figure 1: The arc $\beta$ such that $F_{g-1,2}=\left(F_{g, 1}\right)_{\beta}$, and its close-up $\widetilde{\beta}$

We can now state our main theorem, which is part of Conjecture 6.1 of Church and Farb [3] (more below):

Theorem 1.0.1 Let $g \geq 7$. The image of the map induced by $F_{g-1,1} \rightarrow F_{g, 1}$,

$$
H_{2}\left(\mathcal{I}_{g-1,1} ; \mathbb{Q}\right) \longrightarrow H_{2}\left(\mathcal{I}_{g, 1} ; \mathbb{Q}\right),
$$

generates $H_{2}\left(\mathcal{I}_{g, 1} ; \mathbb{Q}\right)$ as an $\operatorname{Sp}(2 g ; \mathbb{Z})$-module.

As a consequence, $H_{2}\left(\mathcal{I}_{g, 1} ; \mathbb{Q}\right)$ is generated as an $\operatorname{Sp}(2 g, \mathbb{Z})$-module by the image of $H_{2}\left(\mathcal{I}_{6,1} ; \mathbb{Q}\right)$.

We will investigate the group homology of the Torelli group $\mathcal{I}_{g, i}(i=1,2)$ via a spectral sequence for the action of $\mathcal{I}_{g, i}$ on a highly connected complex $B_{*}\left(F_{g, i} ; i\right)$ : Given a $d$-connected complex $X=\left\{X_{p}\right\}_{p \geq 0}$ with a rotation-free action of a group $G$, there is an augmented spectral sequence $E_{p, q}^{*}(X)$ for $p \geq 0$, where

$$
E_{p, q}^{1}(X) \cong \bigoplus_{\sigma \in \Delta_{p-1}} H_{q}\left(G_{\sigma}\right) \Rightarrow 0 \quad \text { for } p+q \leq d+1,
$$

where $G_{\sigma} \subseteq G$ is the stabilizer subgroup for the simplex $\sigma$, and $\Delta_{p}$ denotes a set of representatives for the orbit set $X_{p} / G$. See eg Brown [2, VII, Section 7].

We now define $B_{*}(F ; i)$. First, recall Harer's arc complex $C_{*}(F ; i)$ for $i \in\{1,2\}$ [4]. This is the simplicial complex whose $n$-simplices are $n+1$ isotopy classes of arcs joining two fixed points on $\partial F$ (if $i=1$, the points are on the same boundary component, if $i=2$ they are on different boundary components); the arcs must be disjoint (away from endpoints) and their union must not disconnect $F$. The complex $C_{*}(F ; i)$ has an obvious rotation-free action of $\Gamma(F)$. 
A simplex gives rise to a permutation; namely, given an order of the arcs at the starting point, how the arcs are permuted at the ending point (read off with the opposite orientation, by convention). Then $B_{*}(F ; i)$ is the subcomplex of $C_{*}(F ; i)$ of simplices with the identity permutation. For an illustration, see the left part of Figure 4. Ivanov [5, Theorem 3.5] showed that $B_{*}\left(F_{g, r} ; i\right)$ is $(g-3+i)$-connected for $i=1$, and RandalWilliams [9, Theorem A1] proved the general case.

For $F_{g, 1}$, the action of $\Gamma_{g, 1}$ restricts to an action of $\mathcal{I}_{g, 1}$. For $F_{g-1,2}$, we embed $B_{*}\left(F_{g-1,2} ; 2\right)$ into $B_{*}\left(F_{g, 1} ; 1\right)$ by extending the arcs of each arc simplex in parallel along two fixed disjoint arcs in the pair of pants, as shown in Figure 2. Consequently, $\mathcal{I}_{g-1,2}$ acts on $B_{*}\left(F_{g-1,2} ; 2\right)$.

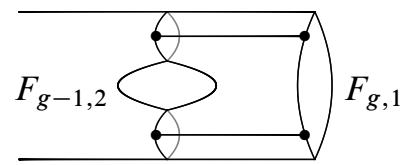

Figure 2: Extending arc simplices $B_{*}\left(F_{g-1,2} ; 2\right) \longrightarrow B_{*}\left(F_{g, 1} ; 1\right)$

Thus we can consider the quotient complex $B_{*}\left(F_{g, 1} ; i\right) / \mathcal{I}_{g, i}$. Concretely, the complex $B_{*}\left(F_{g, 1} ; 1\right) / \mathcal{I}_{g, 1}$ is isomorphic to $\mathcal{B}(g)$, the complex where each $n$-simplex is an ordered basis $\left(x_{0}, x_{1}, \ldots, x_{n}\right)$ for an isotropic summand of $H=H_{1}\left(H_{g, 1} ; \mathbb{Z}\right)$ with respect to the intersection form $i_{\text {alg }}(-,-)$. This isomorphism sends an $\operatorname{arc} \alpha$ to the homology class of the closed curve $\widetilde{\alpha}$ as in Figure 1; see Section 2.1. Similarly, $B_{*}\left(F_{g-1,2} ; 2\right) / \mathcal{I}_{g-1,2}$ is isomorphic to $\mathcal{B}^{a_{1}}(g-1)$, the subcomplex of $\mathcal{B}(g)$ given by those ordered isotropic bases $\left(x_{1}, \ldots, x_{n}\right)$ with $i_{\text {alg }}\left(x_{i}, b_{1}\right)=1$ for all $i=1, \ldots, n$, where $b_{1}$ is part of a fixed standard symplectic basis $\left\{a_{1}, b_{1}, \ldots, a_{g}, b_{g}\right\}$ of $H$.

We can now outline the proof of our main theorem.

Proof of Theorem 1.0.1 The stabilization map $F_{g-1,1} \longrightarrow F_{g, 1}$ is a composition

$$
\alpha \circ \beta: F_{g-1,1} \stackrel{\beta}{\longrightarrow} F_{g-1,2} \stackrel{\alpha}{\longrightarrow} F_{g, 1},
$$

where $\beta$ and $\alpha$ are the maps that glue a pair of pants onto one or two boundary components, respectively.

For $\alpha: F_{g-1,2} \longrightarrow F_{g, 1}$ we use the spectral sequence $E_{p, q}^{r}\left(F_{g, 1} ; 1\right)$ for the action of $\mathcal{I}_{g, 1}$ on the arc complex $B_{*}\left(F_{g, 1} ; 1\right)$. Since $B_{*}\left(F_{g, 1} ; 1\right)$ is $(g-2)$-connected, we obtain

$$
E_{p, q}^{1}\left(F_{g, 1} ; 1\right) \cong \bigoplus_{\alpha \in \Delta_{p-1}} H_{q}\left(\mathcal{I}\left(F_{g, 1}\right)_{\alpha} ; \mathbb{Q}\right) \Rightarrow 0 \quad \text { for } p+q \leq g-1 .
$$


Here, $\Delta_{p}$ denotes a set of representatives for the orbits $B_{p}(F ; 1) / \mathcal{I}(F)$, and $\mathcal{I}(F)_{\alpha}$ is the stabilizer subgroup of $\alpha$ in $\mathcal{I}(F)$. Just as for mapping class groups, one shows that $\mathcal{I}(F)_{\alpha} \cong \mathcal{I}(F) \cap \Gamma\left(F, F_{\alpha}\right)=\mathcal{I}\left(F, F_{\alpha}\right)$, where $F_{\alpha}$ denotes $F$ cut up along $\alpha$. Also, by Proposition 2.1.3(i), $B_{p}\left(F_{g, 1} ; 1\right) / \mathcal{I}_{g, 1} \cong \mathcal{B}(g)^{(p)}$.

Choosing a lift of each simplex gives a map $T: \mathcal{B}(g) \longrightarrow B_{*}\left(F_{g, 1} ; 1\right)$, and we can rewrite (5) as

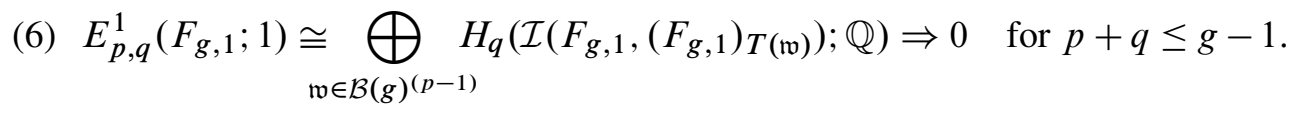

In particular, for $\alpha$ a 0 -simplex, we have $\left(F_{g, 1}\right)_{\alpha} \cong F_{g-1,2}$, and each component map of the differential

$$
d_{2,1}^{1}: \bigoplus_{w \in \mathcal{B}(g)^{(0)}} H_{2}\left(\mathcal{I}\left(F_{g, 1} ;\left(F_{g, 1}\right)_{T(w)}\right) ; \mathbb{Q}\right) \longrightarrow H_{2}\left(\mathcal{I}\left(F_{g, 1}\right) ; \mathbb{Q}\right)
$$

is precisely the map $d_{2,1}^{1}(w): H_{2}\left(\mathcal{I}\left(F_{g, 1} ; F_{g-1,2}\right) ; \mathbb{Q}\right) \longrightarrow H_{2}\left(\mathcal{I}\left(F_{g, 1}\right) ; \mathbb{Q}\right)$ induced by $\alpha$. The differential is $\operatorname{Sp}(2 g, \mathbb{Z})$-equivariant, which can be seen from the construction of $E_{p, q}^{*}\left(F_{g, 1} ; 1\right)$, using the resolution of $\Gamma_{g, 1}$ instead of $\mathcal{I}_{g, 1}$, and applying (1). So since $\operatorname{Sp}(2 g, \mathbb{Z})$ acts transitively on the 0 -simplices $\mathcal{B}(g)^{(0)}$, the image of $d_{2,1}^{1}$ equals the $\operatorname{Sp}(2 g, \mathbb{Z})$-module generated by the image of just one component map $d_{2,1}^{1}(w)=\alpha_{*}$. We will show $d_{2,1}^{1}$ is surjective.

For $\beta: F_{g-1,1} \longrightarrow F_{g-1,2}$, we use the spectral sequence $E_{p, q}^{r}\left(F_{g-1,2} ; 2\right)$ for the action of the Torelli group $\mathcal{I}_{g-1,2}=\mathcal{I}\left(F_{g, 1} ; F_{g-1,2}\right)$ on the arc complex $B_{*}\left(F_{g-1,2} ; 2\right)$. We have $B_{p}\left(F_{g-1,2} ; 2\right) / \mathcal{I}_{g-1,2} \cong \mathcal{B}^{a_{1}}(g-1)$ by Proposition 2.1.3(ii), so in a similar manner as above we obtain

(7) $E_{p, q}^{1}\left(F_{g-1,2} ; 2\right) \cong \bigoplus H_{q}\left(\mathcal{I}\left(F_{g, 1},\left(F_{g-1,2}\right)_{T(\mathfrak{w})}\right) ; \mathbb{Q}\right) \Rightarrow 0$ for $p+q \leq g-1$. $\mathfrak{w} \in \mathcal{B}^{a_{1}}(g)^{(p-1)}$

For a 0 -simplex $\alpha$, we have $\left(F_{g-1,2}\right)_{\alpha} \cong F_{g-1,1}$ and $d_{2,1}^{1}$ has component maps equal to the map induced by $\beta$. The stabilizer subgroup $\operatorname{Sp}(2 g, \mathbb{Z})_{b}$ acts transitively on $\mathcal{B}^{a_{1}}(g-1)^{(0)}$, and $d_{2,1}^{1}$ is $\operatorname{Sp}(2 g, \mathbb{Z})_{b}$-equivariant from (3). Thus the image of $d_{2,1}^{1}$ equals the $\operatorname{Sp}(2 g, \mathbb{Z})_{b}$-module generated by the image of a single component map $\beta_{*}$. We will show $d_{2,1}^{1}$ is surjective.

We see that to prove the main theorem, we must show that the differential $d_{2,1}^{1}$ is surjective in both spectral sequences. To do this, since the spectral sequence converges to zero, it suffices to show that $E_{3,0}^{2}=0$ and $E_{2,1}^{2}=0$.

In Section 2, we show $E_{3,0}^{2}=0$ in both spectral sequences. The main result of the section is: 
Theorem 1.0.2 (i) The quotient complex $B_{*}\left(F_{g, i} ; 1\right) / \mathcal{I}_{g, 1} \cong \mathcal{B}(g)$ is $(g-2)-$ connected.

(ii) The quotient complex $B_{*}\left(F_{g-1, i} ; 2\right) / \mathcal{I}_{g-1,1} \cong \mathcal{B}(g-1)$ is $(g-3)$-connected.

To show $E_{3,0}^{2}=0$, note $E_{p, 0}^{1}\left(F_{g, 1} ; 1\right)=\bigoplus_{\mathfrak{w} \in \mathcal{B}(g)^{(p-1)}} \mathbb{Q}=C_{p-1}(\mathcal{B}(g) ; \mathbb{Q})$ is the $(p-1)$-st chain group of the augmented chain complex for $\mathcal{B}(g)$ with $\mathbb{Q}$-coefficients. Similarly, $E_{p, 0}^{1}\left(F_{g-1,2} ; 2\right)=C_{p-1}\left(\mathcal{B}^{a_{1}}(g-1) ; \mathbb{Q}\right)$. Since $\mathcal{B}(g)$ is $(g-2)$-connected, and $\mathcal{B}^{a_{1}}(g-1)$ is $(g-3)$-connected by Theorem 1.0.2, the homology of the chain complexes is zero in degrees $\leq g-3$. So since $g \geq 7$, we see $E_{3,0}^{2}=0$ in both cases. In Section 3, we show that $E_{2,1}^{2}=0$ in both spectral sequences. This is done via a version of discrete Morse theory, using the concrete description of $E_{*, 1}^{1}$ provided by van den Berg [10].

We briefly mention some accessible improvements of our results: First, extending the result to any number of boundary components, and showing stability for $\mathbb{Z}$-coefficients instead of $\mathbb{Q}$-coefficients; both should be possible by the results of [10]. Second, stability for lower genus, but using [10] would either require improving her results, or only reduce the genus by 1 , since in Theorem 3.0.1, the genus of $S$ must be at least 3 .

We close this introduction by placing the result of the Main Theorem (Theorem 1.0.1) into a larger context: The motivation behind this paper is the question of whether the second Morita-Miller-Mumford class $\kappa_{2} \in H^{4}\left(\Gamma_{g, 1}\right)$ restricts nontrivially to $H^{4}\left(\mathcal{I}_{g, 1}\right)$ or not. One can approach this question by attempting to use the spectral sequence for the fibration (1), for which we must investigate $H^{p}\left(\operatorname{Sp}(2 g ; \mathbb{Z}) ; H^{q}\left(\mathcal{I}_{g, 1}\right)\right)$, we focus here on $q=2$. Such groups have been studied stably by Borel [1], in particular his Theorem 4.4. One way to show that the requirements of the theory are fulfilled would be to show that the $\operatorname{Sp}(2 g, \mathbb{Z})$-representations behave well under the stabilization map $H_{2}\left(\mathcal{I}_{g, 1}\right) \longrightarrow H_{2}\left(\mathcal{I}_{g+1,1}\right)$. The formalization of this is what [3] has termed representation stability, and they conjectured this for $H_{q}\left(\mathcal{I}_{g, 1} ; \mathbb{Q}\right)$.

More precisely, the Main Theorem is basically one of the four conditions (namely, surjectivity) for representation stability of $\mathrm{H}_{2}\left(\mathcal{I}_{g, 1} ; \mathbb{Q}\right)$; the others are injectivity, rationality and stability of the multiplicities of the irreducible representations. We have no results for these three conditions, though injectivity might be solved in a similar manner. Another direction would be to show the Main Theorem for higher homology degrees. Our proof that $E_{2,1}^{2}=0$ is computational and specific to $H_{1}\left(\mathcal{I}_{g, 1}\right)$, and so is not readily generalizable. For the question of $\kappa_{2}$, representation stability for $H_{3}\left(\mathcal{I}_{g, 1}\right)$ would also be needed.

Acknowledgements Boldsen is currently supported by a grant from the Carlsberg Foundation. 


\section{Connectivity of the quotient of the arc complex by the To- relli group}

\subsection{A concrete description of the quotient complexes}

In this section, $H=H_{1}\left(F_{g, 1} ; \mathbb{Z}\right)$. Let $\left\{\alpha_{1}, \beta_{1}, \ldots, \alpha_{g}, \beta_{g}\right\}$ be a standard set of simple closed curves on $F_{g, 1}$ as on Figure 3, with homology classes $a_{i}=\left[\alpha_{i}\right]$ and $b_{i}=\left[\beta_{i}\right]$, such that $\left\{a_{1}, b_{1}, \ldots, a_{g}, b_{g}\right\}$ is a symplectic basis for $H$ with respect to the intersection form $i_{\mathrm{alg}}(-,-)$. Make the convention that the curve $\beta_{1}$ is one of the boundary components of $F_{g-1,2}$.

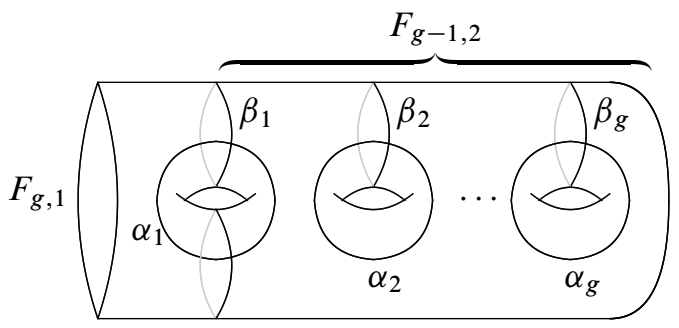

Figure 3: Simple closed curves giving a symplectic basis of $H=H_{1}\left(F_{g, 1}\right)$

Definition 2.1.1 Given a symplectic basis $\left\{a_{1}, b_{1}, \ldots, a_{g}, b_{g}\right\}$ of $H$. For $x \in H$, express $x$ in the basis as $x=\sum_{i=1}^{g}\left(c_{i} a_{i}+d_{i} b_{i}\right)$. Then the $a_{j}$-rank of $x$ is $\mathrm{rk}^{a_{j}}(x):=c_{j}$. Similarly, $\mathrm{rk}^{b_{j}}(x):=d_{j}$.

Note: We have $\mathrm{rk}^{a_{j}}(x)=i_{\mathrm{alg}}\left(x, b_{j}\right)$ and $\mathrm{rk}^{b_{j}}(x)=-i_{\mathrm{alg}}\left(x, a_{j}\right)$.

Definition 2.1.2 $\mathcal{B}(g)$ is the complex where each $n$-simplex is an ordered basis $\left(x_{0}, x_{1}, \ldots, x_{n}\right)$ for an isotropic summand of $H$, ie a summand $V \subseteq H$ where $i_{\text {alg }}(v, w)=0$ for all $v, w \in V$.

Let $\mathcal{B}^{a_{1}}(g-1)$ denote the subcomplex of $\mathcal{B}(g)$ given by those ordered isotropic bases $\left(x_{1}, \ldots, x_{n}\right)$ with $\operatorname{rk}^{a_{1}}\left(x_{i}\right)=1$ for all $i=1, \ldots, n$.

Note, $\mathcal{B}(g)$ and $\mathcal{B}^{a_{1}}(g-1)$ are not simplicial complexes, but a simplex is determined by its vertices and their ordering. More in Section 2.2.

Proposition 2.1.3 [10] (i) The quotient complex $B_{*}\left(F_{g, 1} ; 1\right) / \mathcal{I}_{g, 1}$ is isomorphic to $\mathcal{B}(g)$.

(ii) The quotient complex $B_{*}\left(F_{g-1,2} ; 2\right) / \mathcal{I}_{g-1,2}$ is isomorphic to $\mathcal{B}^{a_{1}}(g-1)$. 
Proof Our original inspiration for this result and its proof was [8, Lemma 6.9], where he showed that the quotient of the complex of simple closed curves in $F_{g, r}$ by the Torelli group, $D_{*}\left(F_{g, r}\right) / \mathcal{I}_{g, r}$, is isomorphic to the simplicial complex $\mathcal{L}(g)$ of lax isotropic bases of $H_{1}\left(F_{g, r}\right)$. But we have later discovered that in her $\mathrm{PhD}$ thesis in 2003, Berg [10, Proposition 2.5.3] has proven the result for $C_{*}\left(F_{g, r} ; i\right) / \mathcal{I}_{g, r}$. We can deduce the Proposition by restricting to $B_{*}\left(F_{g+1-i, i} ; i\right)$, ie requiring that the permutation $=\mathrm{id}$.
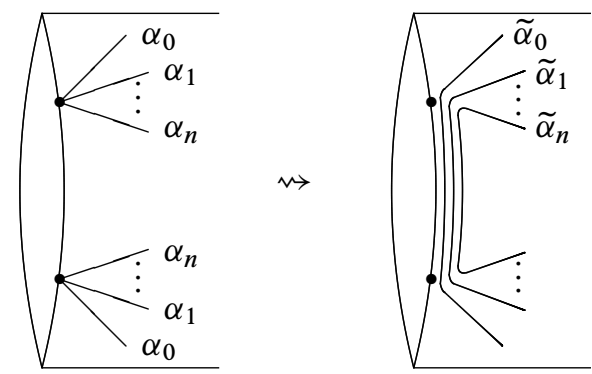

Figure 4: Closing up the arcs of a simplex in $B_{*}\left(F_{g, 1} ; 1\right)$

We briefly mention the map that gives the isomorphism. First consider the map $h: B_{n}\left(F_{g, 1} ; 1\right) \longrightarrow \mathcal{B}(g)$ by

$$
\left(\alpha_{0}, \alpha_{1}, \ldots, \alpha_{n}\right) \mapsto\left(\left[\widetilde{\alpha}_{0}\right],\left[\widetilde{\alpha}_{1}\right], \ldots,\left[\widetilde{\alpha}_{n}\right]\right),
$$

where the simple closed curve $\tilde{\alpha}_{i}$ comes from closing up $\alpha_{i}$ as in Figure 4, and [-] denotes the homology class. The closing-up is always possible, and gives nonintersecting curves, because the arc simplices have permutation id. When we restrict to the subcomplex $B_{*}\left(F_{g-1,1} ; 2\right)$, the target of $h$ is indeed contained in $\mathcal{B}^{a_{1}}(g-1)$; for when closing up an arc $\gamma$ in $B_{*}\left(F_{g, 1} ; 1\right)$ coming from $B_{*}\left(F_{g, 2} ; 2\right)$, the resulting curve $\tilde{\gamma}$ will satisfy $i_{\text {geom }}\left(\tilde{\gamma}, \beta_{1}\right)=1$.

The Torelli group $\mathcal{I}_{g, 1}$ preserves homology classes, so $h$ descends to a map on the quotient $\bar{h}: B_{*}\left(F_{g, 1} ; 1\right) / \mathcal{I}_{g, 1} \longrightarrow \mathcal{B}(g)$. Then what van den Berg proves is that $\bar{h}$ is a bijection.

\subsection{Preliminaries}

2.2.1 Multisimplicial complexes The complexes $\mathcal{B}(g)$ and $\mathcal{B}^{a_{1}}(g)$, along with the other complexes we will define here in Section 2, are of the following type, which we call multisimplicial complexes, for lack of a better word: 
Definition 2.2.1 A nonempty family $\mathcal{K}$ of finite ordered tuples consisting of distinct elements of a universal set $H$ is called a multisimplicial complex if:

(i) for every tuple $\mathfrak{w} \in \mathcal{K}$, and every subtuple $\mathfrak{v}$ of $\mathfrak{w}$, we have $\mathfrak{v} \in \mathcal{K}$. (By a subtuple of $\mathfrak{w}=\left(w_{0}, \ldots, w_{n}\right)$ we mean a tuple $\left(w_{i_{0}}, \ldots, w_{i_{j}}\right)$ where $0 \leq i_{0}<$ $\cdots<i_{j} \leq n$.)

(ii) whether $\mathfrak{w} \in K$ does not depend on the ordering of the tuple $\mathfrak{w}$.

An $(n+1)$-tuple $\mathfrak{w}$ in $\mathcal{K}$ will be called an $n$-simplex. The $i$-th face map $\delta_{i}$ is defined by $\delta_{i}\left(w_{0}, \ldots, w_{n}\right)=\left(w_{0}, \ldots, \widehat{w}_{i}, \ldots, w_{n}\right)$.

Remark 2.2.2 This allows some combinatorial definitions: Let $\mathfrak{v}=\left(v_{0}, \ldots, v_{n}\right)$ and $\mathfrak{w}=\left(w_{0}, \ldots, w_{k}\right)$ be simplices in $\mathcal{K}$. The vertex set of $\mathfrak{w}$ is $V(\mathfrak{w})=\left\{w_{0}, \ldots, w_{k}\right\}$. The link of $\mathfrak{v}, \operatorname{link}_{\mathcal{K}}(\mathfrak{v})$, is defined to be the set of all simplices $\mathfrak{w} \in \mathcal{K}$ such that $\left(v_{0}, \ldots, v_{n}, w_{0}, \ldots, w_{k}\right)$ is a simplex in $\mathcal{K}$. For $\mathfrak{w} \in \operatorname{link}_{\mathcal{K}}(\mathfrak{v})$, we say a simplex $\mathfrak{u}$ is a join of $\mathfrak{v}$ and $\mathfrak{w}$, if $V(\mathfrak{u})=\left\{v_{0}, \ldots, v_{n}, w_{0}, \ldots, w_{k}\right\}$. We will write, by slight abuse of notation, $\mathfrak{u}=\mathfrak{v} * \mathfrak{w}$ to mean that $\mathfrak{u}$ is a join of $\mathfrak{v}$ and $\mathfrak{w}$. Note that $\operatorname{link}_{\mathcal{K}}(\mathfrak{v} * \mathfrak{w})$ is unambiguous.

We are interested in connectivity of multisimplicial complexes, so let $S$ be a simplicial complex, and let $\mathcal{K}$ be a multisimplicial complex, and consider a map $f: S \longrightarrow \mathcal{K}$. We say $f$ is simplicial if $f$ commutes with the face maps.

To say a multisimplicial complex $\mathcal{K}$ is $d$-connected means that the geometric realization, $|\mathcal{K}|$, is $d$-connected. The standard proof for simplicial approximation works equally well to show that a map $f:|S| \longrightarrow|\mathcal{K}|$, where $S$ is a simplicial complex, is homotopic to a simplicial map $g:|S| \longrightarrow|\mathcal{K}|$, that is, $g$ is determined by the map on the underlying complexes, $\tilde{g}: S \longrightarrow \mathcal{K}$. Thus, to show $\mathcal{K}$ is $d$-connected, it suffices to show that for a given simplicial map $f: S^{n} \longrightarrow \mathcal{K}$ where $S^{n}$ is a simplicial $n$-sphere, there exists a simplicial $n$-ball, $B$, with $\partial B=S^{n}$, and a simplicial map $\varphi: B \longrightarrow \mathcal{K}$ with $\left.\varphi\right|_{\partial B}=f$. We now introduce some techniques we will apply to show such connectivity.

Remark 2.2.3 (Gluing simplicial maps) Let $S$ be a simplicial $n$-manifold and $\mathcal{K}$ a multisimplicial complex. Suppose $A, B \subseteq S$ are subcomplexes with $A \cap B=D$ a simplicial (n-1)-submanifold, and we have given simplicial maps $f: A \longrightarrow \mathcal{K}$ and $g: B \longrightarrow \mathcal{K}$. Suppose $\left.f\right|_{D}=\left.g\right|_{D}$. Then for $s \in S$, set

$$
F(s)= \begin{cases}f(s) & \text { if } s \in A, \\ g(s) & \text { if } s \in B .\end{cases}
$$

Then (8) defines a simplicial map $F: S \longrightarrow \mathcal{K}$. 
Definition 2.2.4 (Link move) Let $S$ be a simplicial $n$-manifold, $\mathcal{K}$ a multisimplicial complex, and $f: S \longrightarrow \mathcal{K}$ a simplicial map. Let $\sigma \in S$ be a simplex, and suppose we have a simplicial ball $B$ with $\partial B=\operatorname{link}(\sigma)$ and a simplicial map $\varphi: B \longrightarrow \mathcal{K}$ with $\left.\varphi\right|_{\partial B}=\left.f\right|_{\operatorname{link}(\sigma)}$.
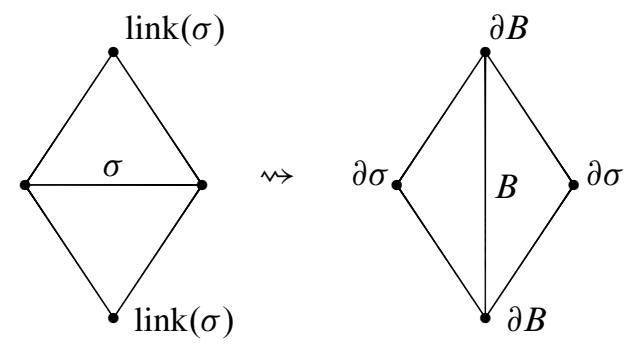

Figure 5: Link move

Let $D=\operatorname{link}(\sigma) * \partial \sigma$. Then $\partial(\operatorname{star}(\sigma))=D=\partial(B * \partial \sigma)$. Define $S^{\prime}=(S \backslash \sigma) \cup_{D} B * \partial \sigma$. Also, define the simplicial map $f^{\prime}: S^{\prime} \longrightarrow \mathcal{K}$, which on a vertex $s \in S^{\prime}$ is given by

$$
f^{\prime}(s)= \begin{cases}\varphi(s) & \text { if } \sigma \in B, \\ f(s) & \text { if } \sigma \notin B,\end{cases}
$$

as in Remark 2.2.3. Since both $\operatorname{star}(\sigma)$ and $B * \partial \sigma$ are faces of $B * \sigma$, there is a homotopy on the geometric realizations from $|f|$ to $\left|f^{\prime}\right|$. We call $f^{\prime}$ the result of performing a link move to $f$ on $\sigma$ with $\varphi$.

2.2.2 ged Now we return to $\mathcal{B}(g)$ and $\mathcal{B}^{a_{1}}(g)$. We define a crucial concept, which we call gcd:

Definition 2.2.5 (gcd) Let $H$ be a free $\mathbb{Z}$ module, and $v_{1}, \ldots, v_{n} \in H$. We define $\operatorname{gcd}\left(v_{1}, \ldots, v_{n}\right)$ to be the divisibility of $v_{1} \wedge \cdots \wedge v_{n}$ in $\wedge^{n} H$, meaning the greatest $m \geq 0$ such that $v_{1} \wedge \cdots \wedge v_{n}=m w$ for some $w \neq 0$ in $\wedge^{n} H$.

For a submodule $W \subset H$, we write $\operatorname{gcd}(W)$ for $\operatorname{gcd}\left(w_{1}, \ldots, w_{k}\right)$, where $w_{1}, \ldots, w_{k}$ is any basis of $W$.

For a single vector $v, \operatorname{gcd}(v)$ is the greatest common divisor of the coefficients when writing $v$ in a basis for $H$, hence the name.

The reason we introduce gcd is that it captures the essence of being a simplex in $\mathcal{B}(g)$ :

Remark 2.2.6 Let $\mathfrak{v}=\left(v_{0}, \ldots, v_{n}\right)$, with $v_{i} \in H=H_{1}\left(F_{g, 1} ; \mathbb{Z}\right)$. Then $\mathfrak{v} \in \mathcal{B}(g)$ if and only if $\left(v_{0}, \ldots, v_{n}\right)$ is isotropic, and $\operatorname{gcd}\left(v_{0}, \ldots, v_{n}\right)=1$. 
Remark 2.2.7 By definition of gcd, we obtain the following properties:

(i) $\operatorname{gcd}\left(v_{1}, \ldots, v_{n}, w_{1}, \ldots, w_{m}\right) \mid \operatorname{gcd}\left(v_{1}, \ldots, v_{n}\right) \cdot \operatorname{gcd}\left(w_{1}, \ldots, w_{m}\right)$.

(ii) If $H=A \oplus B$, and $A^{\prime} \subseteq A, B^{\prime} \subseteq B$ are subsets, then

$$
\operatorname{gcd}\left(A^{\prime}, B^{\prime}\right)=\operatorname{gcd}\left(A^{\prime}\right) \cdot \operatorname{gcd}\left(B^{\prime}\right) \text {. }
$$

For (ii), note that $\bigwedge^{n} A \otimes \bigwedge^{m} B$ is a direct summand of $\bigwedge^{n+m}(A \oplus B)$.

2.2.3 Dual summands In Section 2.5 we need the existence of dual vectors, and we prove all the necessary properties now. First, we define the smallest summand containing a subset:

Definition 2.2.8 For $A \subseteq H$, we define $S(A)$, the smallest summand containing $A$, to be the summand $S(A)=\{x \in H \mid \exists n \in \mathbb{Z} \backslash\{0\}: n x \in\langle A\rangle\}$.

Proposition 2.2.9 Let $H$ be a free $\mathbb{Z}$-module of rank $2 g$ with a symplectic form $i_{\text {alg }}(\cdot, \cdot)$. Given $n \leq g$, and $v_{1}, \ldots, v_{n} \in H$, assume $\operatorname{gcd}\left(v_{1}, \ldots, v_{n}\right)=1$, and set $S=\left\langle v_{1}, \ldots, v_{n}\right\rangle$.

(i) There exists a dual summand $D=D\left(v_{1}, \ldots, v_{n}\right)$ to $S$, meaning $D$ is an isotropic summand of rank $n$, and $D$ has a basis $u_{1}, \ldots, u_{n}$ satisfying

$$
i_{\text {alg }}\left(v_{i}, u_{j}\right)=\delta_{i j}
$$

In particular, $S \oplus D$ is a symplectic summand, so there exists a unique summand $T \subseteq H$, such that $H=(S \oplus D) \oplus T$ is a symplectic splitting.

(ii) Let $k \leq g-n$. Let $D=D\left(v_{1}, \ldots, v_{n}\right)$ be as in (i) be given. Given vectors $w_{1}, \ldots, w_{k}$ with $\operatorname{gcd}\left(w_{1}, \ldots, w_{k}, S\right)=1$, there exists a dual summand $D_{2}$ of $S_{2}=\left\langle v_{1}, \ldots, v_{n}, w_{1}, \ldots, w_{k}\right\rangle$ such that $S \oplus D \subseteq S_{2} \oplus D_{2}$.

(iii) Let $k \leq g-n$. Let $D=D\left(v_{1}, \ldots, v_{n}\right)$ and $T$ be as in (i) be given. Given vectors $w_{1}, \ldots, w_{k}$ with $\operatorname{gcd}\left(w_{1}, \ldots, w_{k}, S, D\right)=1$, there exist a dual summand $D\left(w_{1}, \ldots, w_{k}\right) \subseteq T$

(iv) Let $k \leq g-n-m$. Let $S_{1}, D_{1}, T_{1}$ be as in (i), and let $S_{2}=\left\langle v_{1}, \ldots, v_{n}\right\rangle$ with dual summand $D_{2} \subseteq T_{1}$, also be as in (i). Then given $w_{1}, \ldots, w_{k}$ with $\operatorname{gcd}\left(w_{1}, \ldots, w_{k}, S_{1}, D_{1}, S_{2}\right)=1$, there exists a dual summand $D_{3}=$ $D\left(v_{1}, \ldots, v_{n}, w_{1}, \ldots, w_{k}\right) \subseteq T_{1}$ with $S_{2} \oplus D_{2} \subseteq S_{1} \oplus D_{1} \oplus S_{3} \oplus D_{3}$.

Proof (i) We prove this by induction in $n$. It it not hard to see that one can find $u_{1} \in H$ with $i_{\text {alg }}\left(v_{1}, u_{1}\right)=1$ and $\operatorname{gcd}\left(u_{1}, v_{1}, \ldots, v_{n}\right)=1$. 
Now consider $H_{1}=\left\langle v_{1}, u_{1}\right\rangle^{\perp}$ which gives a symplectic splitting $H=\left\langle v_{1}, u_{1}\right\rangle \oplus H_{1}$. For $n>1$, let $\widetilde{v}_{i}=\operatorname{pr}_{H_{1}}\left(v_{i}\right)$ for $i=2, \ldots, n$. Note that $\operatorname{gcd}\left(\widetilde{v}_{2}, \ldots, \widetilde{v}_{n}\right) \leq_{\text {div }}$ $\operatorname{gcd}\left(u_{1}, v_{1}, v_{2}, \ldots, v_{n}\right)=1$. Then by induction we obtain $u_{2}, \ldots, u_{n}$ and $T$ satisfying the desired properties with respect to $\widetilde{v}_{2}, \ldots, \widetilde{v}_{n}$ in $H_{1}$. We change $u_{1}$ to $\bar{u}_{1}=u_{1}-\sum_{j=2}^{n} c_{j} u_{j}$, where $c_{j}=i_{\text {alg }}\left(v_{i}, u_{1}\right)$. One checks that $D=\left\langle\bar{u}_{1}, u_{2}, \ldots, u_{n}\right\rangle$ is the desired dual summand.

(ii) We have $S, D, T$ given with respect to $v_{1}, \ldots, v_{n}$, as in (i). First we claim there is a rank $2 k$ symplectic summand $W$ in $T$, such that $S \oplus D \oplus W$ contains $w_{1}, \ldots, w_{k}$. To see this, consider the $k$ vectors $\widetilde{w}_{j}=\operatorname{pr}_{T}\left(w_{j}\right)$, and take the smallest summand $S_{W}$ containing them. By (i) we obtain $D_{W}, T_{W}$, where $S_{W} \oplus D_{W}$ is a symplectic summand of rank $\leq 2 k$. If the rank is $<2 k$, add a symplectic summand $R_{W} \subseteq T_{W}$, such that $W=S_{W} \oplus D_{W} \oplus R_{W}$ has rank $2 k$. Next, use (i) on the vectors $v_{1}, \ldots, v_{n}, w_{1}, \ldots, w_{k}$ inside $S \oplus D \oplus W$, yielding a dual summand $S_{2}=S\left(v_{1}, \ldots, v_{n}, w_{1}, \ldots, w_{k}\right)$, and $T_{2}$. Note for dimensional reasons $T_{2}=0$. Thus $S \oplus D \subseteq S \oplus D \oplus W=S_{2} \oplus D_{2}$.

Parts (iii) and (iv) follow easily from (i) and (ii) as we now sketch: To prove (iii), we have $S, D, T$ as in (i). Use (i) on $\widetilde{w}_{j}=\operatorname{pr}_{T}\left(w_{j}\right)$ for $j=1, \ldots, k$, obtaining $D\left(\tilde{w}_{1}, \ldots, \tilde{w}_{k}\right) \subseteq T$. Check this is a dual summand of $\left(w_{1}, \ldots, w_{k}\right)$. To prove (iv), project $\left(v_{1}, \ldots, v_{n}, w_{1}, \ldots, w_{k}\right)$ on $T_{1}$, and call the result $\left(\widetilde{v}_{1}, \ldots, \widetilde{v}_{n}, \widetilde{w}_{1}, \ldots, \widetilde{w}_{k}\right)$. Use (ii) on $\left(\widetilde{w}_{1}, \ldots, \widetilde{w}_{k}\right)$, given $\widetilde{S}_{2}=\left\langle\widetilde{v}_{1}, \ldots, \widetilde{v}_{n}\right\rangle$ and $D_{2}$ in $T_{1}$, to obtain $D_{3}=$ $D\left(\widetilde{v}_{1}, \ldots, \widetilde{v}_{n}, \widetilde{w}_{1}, \ldots, \widetilde{w}_{k}\right)$ with $\widetilde{S}_{2} \oplus D_{2} \subseteq \widetilde{S}_{3} \oplus D_{3} \subseteq T_{1}$. Check $D_{3}$ works.

Remark 2.2.10 Let $n \leq g$. Given $v_{1}, \ldots, v_{n}$ in $H$ with $\operatorname{gcd}\left(v_{1}, \ldots, v_{n}\right)>0$, let $S=S\left(v_{1}, \ldots, v_{n}\right)$ denote the smallest summand containing $\left\langle v_{1}, \ldots, v_{n}\right\rangle$. Then we can choose a basis $v_{1}^{\prime}, \ldots, v_{n}^{\prime}$ for $S$ and get a dual summand $D=D\left(v_{1}^{\prime}, \ldots, v_{n}^{\prime}\right)$. We will call $D$ a dual summand of $S$ (with respect to $v_{1}^{\prime}, \ldots, v_{n}^{\prime}$ ).

\subsection{Connectivity of $B\left(F_{g, 1} ; 1\right) / \mathcal{I}_{g, 1}$}

In this section, $H=H_{1}\left(F_{g, 1} ; \mathbb{Z}\right)$. We prove Theorem 1.0.2 for $i=1$, that the quotient complex $B\left(F_{g, 1} ; 1\right) / \mathcal{I}_{g, 1}$ is $(g-2)$-connected. By Proposition 2.1.3(i), we must show:

Proposition 2.3.1 $\mathcal{B}(g)$ is $(g-2)$-connected.

This follows from Proposition 2.3.3 below by taking $\Delta^{k}=\varnothing$. First we define:

Definition 2.3.2 Let $\Delta^{k} \in \mathcal{B}(g)$ be a $k$-simplex, and write $\mathcal{B}^{\Delta^{k}}(g)=\operatorname{link}_{\mathcal{B}(g)}\left(\Delta^{k}\right)$. If $k=-1$, we take $\Delta^{k}=\varnothing$ and $\mathcal{B}^{\Delta^{k}}(g)=\mathcal{B}(g)$.

Let $W \subseteq H$ be a $\mathbb{Z}$-subgroup, and define $\mathcal{B}^{\Delta^{k} ; W}(g)$ to be the subcomplex of $\mathcal{B}^{\Delta^{k}}(g)$ consisting of the simplices whose vertices are in $W$. 
Proposition 2.3.3 Let $g \geq 1$ and $-1 \leq k<g$. Let $\Delta^{k}$ be a $k-\operatorname{simplex}$ in $\mathcal{B}(g)$. Then $\mathcal{B}^{\Delta^{k}}(g)$ is $(g-k-3)$-connected.

This follows from the Lemma below, the proof of which is modeled on [8, Proposition 6.13], but Putman's argument has a gap, which we repair.

Lemma 2.3.4 For $g \geq 1$, fix $-1 \leq k<g$. Let $\Delta^{k}$ be a $k$-simplex in $\mathcal{B}(g)$. Then for all vectors $x \in \mathcal{B}^{\Delta^{k}}(g)$, the following hold.

(i) For $-1 \leq n \leq g-k-3$, we have $\pi_{n}\left(\mathcal{B}^{\Delta^{k}} ;\langle x\rangle^{\perp}(g)\right)=0$.

(ii) For $-1 \leq n \leq g-k-3$, we have $\pi_{n}\left(\mathcal{B}^{\Delta^{k}}(g)\right)=0$.

Proof We first prove (i). Assume inductively that $\pi_{n^{\prime}}\left(\mathcal{B}^{\Delta^{k^{\prime}} ;\langle x\rangle^{\perp}}(g)\right)=0$ and $\pi_{n^{\prime}}\left(\mathcal{B}^{\Delta_{k^{\prime}}}(g)\right)=0$ for all $n^{\prime}<n$ and all $\Delta^{k^{\prime}}$ such that $n^{\prime} \leq g-k^{\prime}-3$. The case $n=-1$ holds, since $x \in \mathcal{B}^{\Delta^{k} ;\langle x\rangle^{\perp}}(g) \neq \varnothing$ for $k<g$.

So let $n \geq 0$, and let $S$ be a simplicial $n$-sphere and $f: S \longrightarrow \mathcal{B}^{\Delta^{k} ;\langle x\rangle^{\perp}}(g)$ a simplicial map. We seek a simplicial $n$-ball $B$ with $\partial B=S$ and a simplicial map $F: B \longrightarrow \mathcal{B}^{\Delta^{k}} ;\langle x\rangle^{\perp}(g)$ such that $\left.F\right|_{S}=f$.

Fix a symplectic basis $\mathfrak{X}$ of $H$ extending the isotropic basis $\left(\Delta^{k}, x\right)$, use this basis to define $\mathrm{rk}^{x}$ as in Definition 2.1.1, and consider

$$
R=R_{x}=\max \left\{\left|\operatorname{rk}^{x}(\varphi(s))\right| \mid s \in S^{(0)}\right\} .
$$

If $R=0$, any $v \in f(S)$ has $\operatorname{rk}^{x}(v)=0$, and thus $f(S) \subseteq \operatorname{link}_{\mathcal{B}^{\Delta^{k}} ;\langle x\rangle^{\perp}}(x)$. So we can take the simplicial $n$-ball $B=S *+$ (where + denotes a new vertex) and define a simplicial map $F: B \longrightarrow \mathcal{B}^{\Delta^{k} ;\langle x\rangle^{\perp}}(g)$ by $F(+)=x$, as in Remark 2.2.3. This proves the result for $R=0$.

Now assume that $R>0$, and call $\sigma \in S$ regularly bad if all vertices $s$ of $\sigma$ satisfy $\left|\mathrm{rk}^{x}(\varphi(s))\right|=R$. Let $\sigma$ be a regularly bad simplex of maximal dimension, say $\operatorname{dim} \sigma=m$. By maximality of $\sigma, f(\operatorname{link}(\sigma)) \subseteq \operatorname{link}(f(\sigma))$, and thus we have a map

$$
\left.f\right|_{\operatorname{link}(\sigma)}: \operatorname{link}_{S}(\sigma) \longrightarrow \mathcal{B}^{\Delta^{k} * f(\sigma) ;\langle x\rangle^{\perp}}(g) .
$$

Here, $\operatorname{link}_{S}(\sigma)$ is a simplicial $(n-m-1)-$ sphere, and the goal is to obtain a simplicial $(n-m)$-ball $D$ with $\partial D=\operatorname{link}_{S}(\sigma)$ and a simplicial map

$$
\varphi: D \longrightarrow \mathcal{B}^{\Delta^{k} * f(\sigma) ;\langle x\rangle^{\perp}}(g) \quad \text { with }\left.\varphi\right|_{\partial D}=\left.f\right|_{\operatorname{link}(\sigma)} .
$$

This follows from the inductive hypothesis if $x \in \mathcal{B}^{\Delta^{k} * f(\sigma)}(g)$. But this might not be the case, so assume $x \notin \mathcal{B}^{\Delta^{k} * f(\sigma)}(g)$, in other words $\operatorname{gcd}\left(x, f(\sigma), \Delta^{k}\right) \neq 1$. (This is what is missing in Putman's argument). 
There are two possibilities. The first is $\operatorname{gcd}\left(x, f(\sigma), \Delta^{k}\right)>1$. In this case, the smallest summand $V$ containing $\left\langle x, f(\sigma), \Delta^{k}\right\rangle$ has rank $1+(\operatorname{dim} f(\sigma)+1)+(k+1)$, and so we can choose a basis for $V$ of the form $\left\{\tilde{x}, f(\sigma), \Delta^{k}\right\}$. Since $V$ is isotropic, we get

$$
\mathcal{B}^{\Delta^{k} * f(\sigma) ;\langle x\rangle^{\perp}}(g)=\mathcal{B}^{\Delta^{k} * f(\sigma) ;\langle\tilde{x}\rangle^{\perp}}(g) .
$$

Now by construction, $\tilde{x} \in \mathcal{B}^{\Delta^{k} * f(\sigma)}(g)$, so we get (11) by induction.

The second possibility is $\operatorname{gcd}\left(x, f(\sigma), \Delta^{k}\right)=0$. Then $V=\left\langle f(\sigma), \Delta^{k}\right\rangle$ is a summand, and $x \in V$. Choose a basis of $V$ extending $x$, ie $\left\{x, w_{0}, \ldots, w_{\ell}\right\}$, such that $\operatorname{rk}^{x}\left(w_{i}\right)=0$. Note $\ell=\operatorname{dim}(f(\sigma))+k$, and $\mathfrak{w}=\left(w_{0}, \ldots, w_{\ell}\right) \in \mathcal{B}(g)$. Then

$$
\mathcal{B}^{\Delta^{k} * f(\sigma) ;\langle x\rangle^{\perp}}(g)=\mathcal{B}^{x * \mathfrak{w} ;\langle x\rangle^{\perp}}(g) .
$$

Let $y$ denote the basis vector in $\mathfrak{X}$ dual to $x$, ie $i_{\text {alg }}(x, y)=1$. Consider

$$
\text { pr: }\langle x\rangle^{\perp} \longrightarrow\langle x, y\rangle^{\perp}, \quad \operatorname{pr}(h)=h-i_{\text {alg }}(h, y) x .
$$

This can be extended to a map on simplices, which we call pr again, by using pr on each vertex. Then for $\mathfrak{v} \in \mathcal{B}^{x * \mathfrak{w},\langle x\rangle^{\perp}}(g)$ we get that $\operatorname{pr}(\mathfrak{v}) \in \mathcal{B}^{x * \mathfrak{w},\langle x, y\rangle^{\perp}}(g)$, from (13). We can identify $\langle x, y\rangle^{\perp}$ with $\left\langle a_{1}, b_{1}, \ldots, a_{g-1}, b_{g-1}\right\rangle$, and since $w_{0}, \ldots, w_{\ell} \in\langle x, y\rangle^{\perp}$, this identification turns pr into a map

$$
\operatorname{pr}: \mathcal{B}^{x * \mathfrak{w},\langle x\rangle^{\perp}}(g) \longrightarrow \mathcal{B}^{\mathfrak{w}}(g-1) .
$$

We then consider the composition pr $\left.\circ f\right|_{\operatorname{link}(\sigma)}$, and get by induction in (ii) that there is a simplicial ball $D$ with $\partial D=\operatorname{link}(\sigma)$ and a simplicial map $\tilde{\varphi}$ such that the left-hand square commutes in the following diagram:

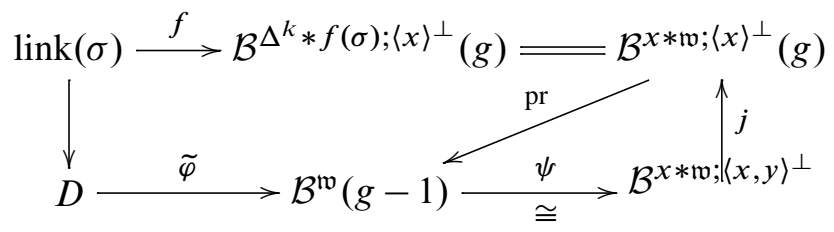

Here, $j$ is induced by the subspace inclusion $\langle x, y\rangle^{\perp} \hookrightarrow H$, and $\operatorname{pr} \circ j \circ \psi=\mathrm{id}$. We modify $\tilde{\varphi}$ to a map $\varphi: D \longrightarrow \mathcal{B}^{\Delta^{k} * f(\sigma)}(g)$ satisfying (11) via

$$
\varphi(s)= \begin{cases}f(s) & \text { if } s \in \operatorname{link}(\sigma), \\ j \circ \psi \circ \widetilde{\varphi}(s) & \text { if } s \in D \backslash \partial D,\end{cases}
$$

and Remark 2.2.3. (To show $\varphi$ is well-defined, use $\tilde{\varphi}=\operatorname{pr} \circ \varphi$.) This shows we have $\varphi$ as in (11).

We now modify $\varphi$ to a map $\varphi^{\prime}$ with the following property:

$$
\left|\mathrm{rk}^{x}\left(\varphi^{\prime}(s)\right)\right|<R \quad \text { for all } s \in D^{(0)} .
$$


We obtain $\varphi^{\prime}$ by performing division with remainder, as in [8]: Let $t \in \sigma$ be fixed, set $v=\varphi(t)$. By division we obtain $q_{s} \in \mathbb{Z}$ such that $\left|\operatorname{rk}^{x}\left(\varphi(s)-q_{s} v\right)\right|<\left|\operatorname{rk}^{x}(v)\right|=R$ for all $s \in D^{(0)}$. For $s \in \partial D=\operatorname{link}(\sigma)$ we take $q_{s}=0$. We then set $\varphi^{\prime}(s)=\varphi(s)-q_{s} v$ for $s \in D^{(0)}$. By Remark 2.2.3 we get a simplicial map $\varphi^{\prime}: D \longrightarrow \mathcal{B}^{\Delta^{k} * f(\sigma),\langle x\rangle^{\perp}}(g)$ with $\left|\operatorname{rk}^{x}\left(\varphi^{\prime}(s)\right)\right|<R$ for all $s \in D^{(0)}$, as desired.

Then we do a link move to $f$ on $\sigma$ with $\varphi^{\prime}$ (see Definition 2.2.4), which produces a map homotopic to $f$, removing $\sigma$. Continuing this process inductively in the maximal dimension of regularly bad simplices, we can obtain $R=0$, so we are done.

We next prove (ii). This is done in a similar manner, but instead of $R_{x}$ we use $R_{y}$, where again $y$ is the dual basis vector to $x$. For $R_{y}=0$, the image of the sphere $S$ is contained in $\langle x\rangle^{\perp}$, so part (i) applies. For $R_{y}>0$, we remove bad simplices precisely as above, which is easier since the analogue of (10) now directly implies (11).

\subsection{Connectivity of $B\left(F_{g, 2} ; 2\right) / \mathcal{I}_{g, 2}$, first part}

In this section, $H=H(g+1)=H_{1}\left(F_{g+1,1} ; \mathbb{Z}\right)$. We prove Theorem 1.0.2 for $i=2$ : The quotient complex $B\left(F_{g, 2} ; 2\right) / \mathcal{I}_{g, 2}$ is $(g-2)$-connected. By Proposition 2.1.3(ii), to prove this we must show:

Theorem 2.4.1 $\mathcal{B}^{a_{1}}(g)$ is $(g-2)$-connected.

Definition 2.4.2 For a vector $v \in H$, let $\operatorname{pr}_{2}(v)$ denote the projection of $v$ onto the subspace $\left\langle a_{2}, b_{2}, \ldots, a_{g+1}, b_{g+1}\right\rangle$. For a simplex $\mathfrak{v}=\left(v_{1}, \ldots, v_{n}\right) \in \mathcal{B}^{a_{1}}(g)$, let $\operatorname{pr}_{2}(\mathfrak{v})=\left(\operatorname{pr}_{2}\left(v_{1}\right), \ldots, \operatorname{pr}_{2}\left(v_{n}\right)\right)$.

Note that $\operatorname{pr}_{2}(\mathfrak{v})$ is in general not a simplex.

The basic idea behind the proof of connectivity of $\mathcal{B}^{a_{1}}(g)$ is the following: Because the $a_{1}$-coordinate in a simplex $\mathfrak{v} \in \mathcal{B}^{a_{1}}(g)$ is fixed to be 1 , we cannot manipulate the vectors of $\mathfrak{v}$ as we did by using division with remainder in the proof for $\mathcal{B}(g)$. We take two major steps be able to ignore the $a_{1}-$ and $b_{1}$-coordinates of $\mathfrak{v}$ : In Section 2.4, we reduce to the case where the $b_{1}$-coordinate is fixed, and the projection $\operatorname{pr}_{2}(\mathfrak{v})$, form a simplex in $\mathcal{B}(g)$. Section 2.5 is then dedicated to adapting the proof of Proposition 2.3.3 to the new situation.

For a simplex $\mathfrak{v} \in \mathcal{B}^{a_{1}}(g)$, we will often need the projection map $\mathrm{pr}_{2}$ in connection with gcd (see Definition 2.2.5), so we introduce the following notation:

Definition 2.4.3 $\operatorname{gcd}_{2}(\mathfrak{v})=\operatorname{gcd}\left(\operatorname{pr}_{2}(\mathfrak{v})\right)$. 
We recall from Remark 2.2.7(i) if $\mathfrak{v}, \mathfrak{w} \in \mathcal{B}^{a_{1}}(g)$ and $\mathfrak{v} * \mathfrak{w}$ is a simplex, then

$$
\operatorname{gcd}_{2}(\mathfrak{v} * \mathfrak{w}) \mid \operatorname{gcd}_{2}(\mathfrak{v}) \operatorname{gcd}_{2}(\mathfrak{w}) .
$$

From now on, for $\mathfrak{v} \in \mathcal{B}^{a_{1}}(g)$ (Sections 2.4, 2.5, and 3.6) we write $S(\mathfrak{v})=S\left(\operatorname{pr}_{2}(\mathfrak{v})\right.$ ); see Definition 2.2.8.

Definition 2.4.4 Let $\Delta^{k} \in \mathcal{B}^{a_{1}}(g)$. Write $\mathcal{B}^{a_{1}, \Delta^{k}}(g)=\operatorname{link}_{\mathcal{B}^{a_{1}}(g)}\left(\Delta^{k}\right)$.

- Let $\mathcal{B}_{\mathrm{gcd} \neq 0}^{a_{1}}(g)$ be the subcomplex of $\mathcal{B}^{a_{1}}(g)$ consisting of simplices $\mathfrak{v}$ satisfying $\operatorname{gcd}_{2}(\mathfrak{v}) \neq 0$.

- Let $\mathcal{B}_{\text {gcd }}^{a_{1}, \Delta_{1}^{k}}(g)$ be the subcomplex of $\mathcal{B}^{a_{1}, \Delta^{k}}(g)$ consisting of simplices $\mathfrak{v}$ satisfying $\operatorname{gcd}_{2}\left(\mathfrak{v} ; S\left(\Delta^{k}\right)\right)=1$.

- Let $t \in \mathbb{Z}$. Define $\mathcal{B}_{\mathrm{gcd}}^{a_{1}, \Delta_{1 ; t}^{k}}(g)$ to be the subcomplex of $\mathcal{B}_{\mathrm{gcd}}^{a_{1}, \Delta_{1}^{k}}(g)$ consisting of simplices $\left(v_{1}, \ldots, v_{n}\right)$ where $\mathrm{rk}^{b_{1}}\left(v_{i}\right)=t$ for all $i$.

Remark 2.4.5 If $\operatorname{gcd}_{2}\left(\mathfrak{v} ; S\left(\Delta^{k}\right)\right)=1$, the inequality (17) implies $\operatorname{gcd}_{2}\left(\mathfrak{w} ; S\left(\Delta^{j}\right)\right)=1$ for all subsimplices $\Delta^{j} \subseteq \Delta^{k}$ and all subsimplices $\mathfrak{w} \subseteq \mathfrak{v}$.

We first consider what happens when $\operatorname{gcd}_{2}\left(\Delta^{k}\right)=0$.

Lemma 2.4.6 Let $\Delta^{k}$ be a $k$-simplex in $\mathcal{B}^{a_{1}}(g)$ with $\operatorname{gcd}_{2}\left(\Delta^{k}\right)=0$. Then $\mathcal{B}^{a_{1}, \Delta^{k}}(g)$ is $(g-k-2)-$ connected.

Proof Let $\Delta^{k}=\left(v_{0}, \ldots, v_{k}\right)$ and denote $\operatorname{pr}_{2}\left(\Delta^{k}\right)$ by $\left(\widetilde{v}_{0}, \ldots, \widetilde{v}_{k}\right)$, that is, $v_{i}=$ $a_{1}+r_{i} b_{1}+\tilde{v}_{i}, i=0, \ldots, k$. Since $\operatorname{gcd}_{2}\left(\Delta^{k}\right)=0$, the set $\left\{\tilde{v}_{0}, \ldots, \tilde{v}_{k}\right\}$ is linearly dependent, which gives some $c_{0}, \ldots, c_{k} \in \mathbb{Z}$ relatively prime, with

$$
\sum_{i=0}^{k} c_{i} v_{i}=s a_{1}+t b_{1} \quad \text { for some } s, t \in \mathbb{Z} .
$$

Since $\left\{v_{0}, \ldots, v_{k}\right\}$ is isotropic, $0=i_{\text {alg }}\left(v_{i}, s a_{1}+t b_{1}\right)=t-s r_{i}$ for all $i=0, \ldots, k$. Thus all $r_{i}$ have a common value, $r=t / s$, and $\mathrm{rk}^{b_{1}}\left(v_{i}\right)=r_{i}=r$ for $i=0, \ldots, k$. Using (18) it is easy to conclude that for any $\mathfrak{w} \in \mathcal{B}^{a_{1}, \Delta^{k}}(g)$, the $b_{1}$-coordinate of each vertex in $\mathfrak{w}$ is always $r$.

Write $H_{2}:=\operatorname{pr}_{2}(H)=\left\langle a_{2}, b_{2}, \ldots, a_{g+1}, b_{g+1}\right\rangle$. Let $\left(\tilde{x}_{1}, \ldots, \tilde{x}_{k}\right)$ be a basis of $\left\langle\widetilde{v}_{0}, \ldots, \widetilde{v}_{k}\right\rangle$ in $H_{2}$; then set $x_{0}=a_{1}+r b_{1}$, and $x_{i}=x_{0}+\tilde{x}_{i}$. Then $\Lambda^{k}=$ $\left(x_{0}, x_{1}, \ldots, x_{k}\right)$ is also a simplex in $\mathcal{B}^{a_{1}}$, and $\mathcal{B}^{a_{1}, \Lambda^{k}}(g)=\mathcal{B}^{a_{1}, \Delta^{k}}(g)$.

If we identify $H_{2}$ with $H(g)$, then we see that $\tilde{\Lambda}^{k-1}:=\left(\tilde{x}_{1}, \ldots, \tilde{x}_{k}\right)$ becomes a $(k-1)$-simplex in $\mathcal{B}(g)$. Then $\mathcal{B}^{a_{1}, \Lambda^{k}}(g) \cong \mathcal{B}^{\widetilde{\Lambda}^{k-1}}(g)$ via the isomorphism $\mathfrak{v} \mapsto$ $\operatorname{pr}_{2}(\mathfrak{v})$, since $\mathfrak{v}=a_{1}+r b_{1}+\operatorname{pr}_{2}(\mathfrak{v})$ by the above. From Proposition 2.3.3 we know $\mathcal{B}^{\Lambda^{k-1}}(g)$ is $(g-k-2)-$ connected. 
Consequently, we can focus on simplices $\Delta^{k} \in \mathcal{B}^{a_{1}}(g)$ with $\operatorname{gcd}_{2}\left(\Delta^{k}\right) \neq 0$ :

Proposition 2.4.7 Let $g \geq 3$ and $-1 \leq k \leq g-1$. For any $k$-simplex $\Delta^{k} \in \mathcal{B}^{a_{1}}(g)$ with $\operatorname{gcd}_{2}\left(\Delta^{k}\right) \neq 0$, consider the following:

(i) $\pi_{n}\left(\mathcal{B}^{a_{1}}(g)\right)=0$ for $-1 \leq n \leq g-2$.

(ii) $\pi_{n}\left(\mathcal{B}_{\operatorname{gcd} \neq 0}^{a_{1}}(g)\right)=0$ for $-1 \leq n \leq g-2$.

(iii) $\pi_{n}\left(\mathcal{B}_{\mathrm{gcd}}^{a_{1}, \Delta_{1}^{k}}(g)\right)=0$ for $-1 \leq n \leq g-k-3$.

(iv) $\pi_{n}\left(\mathcal{B}_{\text {gcd }=1 ; t}^{a_{1}, \Delta^{k}}(g)\right)=0$ for $-1 \leq n \leq g-k-3$, where

$$
t= \begin{cases}0 & \text { if } \operatorname{gcd}_{2}\left(\Delta^{k}\right)=1, \\ \operatorname{rk}^{b_{1}}\left(v_{0}\right) & \text { if } \operatorname{gcd}_{2}\left(\Delta^{k}\right) \neq 1, \text { where } \Delta^{k}=\left(v_{0}, \ldots, v_{k}\right) .\end{cases}
$$

Then (iv) $\Rightarrow$ (iii) $\Rightarrow$ (ii) $\Rightarrow$ (i) .

Proof All the implications $\Rightarrow$ will be shown similarly, so we give the first one in detail, and in the others focus on the differences.

(iv) $\Rightarrow$ (iii) Assume $-1 \leq n \leq g-k-3$. Let $S$ be a simplicial $n-$ sphere, and let $f: S \longrightarrow \mathcal{B}_{\mathrm{gcd}=1}^{a_{1} \Delta^{k}}(g)$ be a simplicial map. We wish to homotope $f$ through a series of link moves (possibly subdividing $S$ ), so in the end $f(S)$ lies in $\mathcal{B}_{\text {gcd }}^{a_{1}, \Delta_{1}^{k}}(g)$.

Let $t$ be as specified in (19). Call a simplex $\sigma \in S$ regularly bad, if all $b_{1}$-coordinates in $f(\sigma)$ are $\neq t$, ie $f(\sigma)$ is disjoint from $\mathcal{B}_{\mathrm{gcd}=1 ; t}^{a_{1}, \Delta^{k}}(g)$. Let $\sigma \in S$ be regularly bad of maximal dimension, say $\operatorname{dim}(\sigma)=m$.

We claim $f(\operatorname{link}(\sigma)) \subseteq \operatorname{link}(f(\sigma))$. Since $f$ is simplicial, it suffices to show that $f(\sigma) \cap f(\operatorname{link}(\sigma))=\varnothing$. This follows from the fact that $\sigma$ is regularly bad of maximal dimension; indeed if not, and $v \in f(\sigma) \cap f(\operatorname{link}(\sigma))$ is a vertex, then $v=f(s)$ for $s \in S^{(0)}$, and $s * \sigma$ would also be regularly bad, contradicting the maximality of $\sigma$. This argument is quite general (it holds for most definitions of regularly bad we will use) and the result will henceforth be used without comment.

It follows that every simplex $\mathfrak{v} \in f(\operatorname{link}(\sigma))$ has the property that all $b_{1}$-coordinates of $\mathfrak{v}$ are $t$. So

$$
\left.f\right|_{\operatorname{link}(\sigma)}: \operatorname{link}(\sigma) \longrightarrow \mathcal{B}_{\mathrm{gcd}=1 ; t}^{a_{1}, f(\sigma) * \Delta^{k}}(g),
$$

and we know from (iv), since $\operatorname{dim}\left(f(\sigma) * \Delta^{k}\right) \leq k+m+1$, that $\mathcal{B}_{\mathrm{gcd}}^{a_{1}, f(\sigma) * \Delta^{k}}(g)$ is $(g-k-m-2)-$ connected. Also, $\operatorname{link}(\sigma)$ is an $(n-m-1)-$ sphere, where $n-m-1 \leq$ $g-k-m-2$. So there is a simplicial $(n-m)-$ ball $B$ with $\partial B=\operatorname{link}(\tau)$, and a map $\varphi: B \longrightarrow \mathcal{B}^{a_{1}, \Delta^{k} * \mathfrak{w}}(g)$, such that $\left.\varphi\right|_{\partial B}=\left.f\right|_{\operatorname{link}(\tau)}$. Now we perform a link move to $f$ 
on $\tau$ with $\varphi$. Call the resulting map $f^{\prime}$; it is homotopic to $f$. Note, all this follows from (20) and the induction in (iv).

We wish to show that we have introduced no new regularly bad simplices in $S^{\prime}$ of dimension $\geq m$. By construction a new simplex in $S^{\prime}$ has the form $\tau_{1} * \tau_{2}$, where $\tau_{1} \in \partial \sigma$ and $\tau_{2} \in B$ (one of them can be the empty simplex). Thus $f\left(\tau_{2}\right)$ has all $b_{1}$-coordinates equal to $t$, and so if $\tau_{2} \neq \varnothing$, then $\tau_{1} * \tau_{2}$ cannot be regularly bad. So we have introduced no new regularly bad simplices.

This shows we can, through homotopies of the starting map $f$, remove all bad simplices by induction in the maximal dimension of regularly bad simplices. When there are no regularly bad simplices left, we have $f: S \longrightarrow \mathcal{B}_{\mathrm{gcd}=1 ; t}^{a_{1}, \Delta^{k}}(g)$, and by (iv) this complex is $(g-k-3)-$ connected, so we are done.

(iii) $\Rightarrow$ (ii) Let $S$ be a simplicial $n$-sphere, and let $f: S \longrightarrow \mathcal{B}_{\mathrm{gcd} \neq 0}^{a_{1}}(g)$ be a simplicial map. We say $\sigma \in S$ is regularly bad if for all vertices $v \in f(\sigma)$ we have $\operatorname{gcd}_{2}(v, S(f(\sigma) \backslash v))>1$. Here $f(\sigma) \backslash v$ denotes the difference in vertex sets. Let $\sigma \in S$ be regularly bad of maximal dimension.

We claim

$$
\left.f\right|_{\operatorname{link}(\sigma)}: \operatorname{link}(\sigma) \longrightarrow \mathcal{B}_{\mathrm{gcd}=1}^{a_{1}, f(\sigma)}(g) .
$$

By maximality, $f(\operatorname{link}(\sigma)) \subseteq \operatorname{link}(f(\sigma))$. So we must show for all $\tau \subseteq \operatorname{link}(\sigma)$ that $\operatorname{gcd}_{2}(f(\tau), S(f(\sigma)))=1$. Assume for contradiction there is $\tau \subseteq \operatorname{link}(\sigma)$ such that $\operatorname{gcd}_{2}(f(\tau), S(f(\sigma)))>1$. We know $\tau * \sigma$ is not regularly bad by maximality of $\sigma$, so there is a vertex $v \in f(\tau) * f(\sigma)$, such that

$$
\operatorname{gcd}_{2}(v, S(f(\tau) * f(\sigma) \backslash v))=1 .
$$

If $v \in f(\sigma)$ then we get by Remark 2.4.5,

$$
1=\operatorname{gcd}_{2}(v, S(f(\tau) * f(\sigma) \backslash v))=\operatorname{gcd}_{2}(v, S(f(\sigma) \backslash v))>1 .
$$

So we know that $v \in f(\tau)$. Consider $f(\tau) \backslash v$. We see from (22) that

$\operatorname{gcd}_{2}(f(\tau) \backslash v, S(f(\sigma)))=\operatorname{gcd}_{2}(v, f(\tau) \backslash v, S(f(\sigma)))=\operatorname{gcd}_{2}(f(\tau), S(f(\sigma))) \neq 1$.

Thus we can use the same argument with $f(\tau) \backslash v$ instead of $f(\tau)$. Iterating this, we reach the absurd conclusion that $\operatorname{gcd}_{2}(S(f(\sigma))) \neq 1$, so we have shown the claim (21).

Now the proof runs as above by induction in (iii). When there are no regularly bad simplices left, we have $f: S \longrightarrow \mathcal{B}_{\text {gcd }}^{a_{1}, \Delta_{1}^{k}}(g)$, so we are done.

(ii) $\Rightarrow$ (i) A simplex $\sigma \in S$ is called regularly bad if it satisfies both $\operatorname{gcd}_{2}(f(\sigma))=0$, and $\operatorname{gcd}_{2}(\mathfrak{v}) \neq 0$ for all proper subsimplices $\mathfrak{v} \subsetneq f(\sigma)$. Let $\sigma$ be regularly bad 
of maximal dimension, say $\operatorname{dim}(\sigma)=m$. Then $f(\operatorname{link}(\sigma)) \subseteq \operatorname{link}(f(\sigma))$, and by Lemma 2.4.6, $\operatorname{link}_{\mathcal{B}^{a}}{ }_{1}(g)(f(\sigma))=\mathcal{B}^{a_{1}, f(\sigma)}(g)$ is at least $(g-m-2)$-connected. Using this instead of induction yields the result.

\subsection{Connectivity of $B\left(F_{g, 2} ; 2\right) / \mathcal{I}_{g, 2}$, second part}

In this section we prove the connectivity of $\mathcal{B}_{\mathrm{gcd}=1 ; t}^{a_{1} \Delta^{k}}(g)$, where $t \in \mathbb{Z}$ is as in (19). This turns out to be trickier than one should think, and we need more reductions to prove the result. The problem is that $\Delta^{k}$ itself need neither satisfy $\operatorname{gcd}_{2}\left(\Delta^{k}\right)=1$ nor that $\mathrm{rk}^{b_{1}}(v)=t$ for all vertices $v$ of $\Delta^{k}$.

In this section, recall the meaning of $\Delta=\Delta_{1} * \Delta_{2}$ etc, from Remark 2.2.2.

Remark 2.5.1 We will apply Proposition 2.2.9 to the projection simplices, and use the following notation: If $\Delta$ is an $n$-simplex, we will write $S(\Delta)=S\left(\operatorname{pr}_{2}(\Delta)\right)$, the smallest summand containing $\operatorname{pr}_{2}(\Delta)$. Then $D(\Delta)$ denotes a dual summand of $S(\Delta)$ in $H_{2}=\operatorname{pr}_{2}(H)$, and $T(\Delta)$ denotes the symplectic subspace such that $S(\Delta) \oplus D(\Delta) \oplus T(\Delta)=H_{2}$. Then (iii) and (iv) of Proposition 2.2.9 can be stated as follows:

(i) Given $\Delta_{1}$ and $\Delta_{2}$ such that $\Delta_{1} * \Delta_{2}$ is a simplex with $\operatorname{gcd}_{2}\left(\Delta_{1} * \Delta_{2}\right) \neq 0$, and given a dual summand $D\left(\Delta_{1}\right)$. If $\operatorname{gcd}_{2}\left(\Delta_{2}, S\left(\Delta_{1}\right), D\left(\Delta_{1}\right)\right)=1$, then there is $D\left(\Delta_{2}\right) \subseteq T\left(\Delta_{1}\right)$. In particular we can choose $D\left(\Delta_{1} * \Delta_{2}\right)=D\left(\Delta_{1}\right) \oplus D\left(\Delta_{2}\right)$.

(ii) Given $\Delta_{1}, \Delta_{2}$ and $\Delta_{3}$ such that $\Delta_{1} * \Delta_{2} * \Delta_{3}$ is a simplex, and given dual summands $D\left(\Delta_{1}\right)$ and $D\left(\Delta_{2}\right)$. If $\operatorname{gcd}_{2}\left(\Delta_{2} * \Delta_{3}, S\left(\Delta_{1}\right), D\left(\Delta_{1}\right)\right)=1$, then there is $D\left(\Delta_{2} * \Delta_{3}\right) \subseteq T\left(\Delta_{1}\right)$ with

$$
S\left(\Delta_{2}\right) \oplus D\left(\Delta_{2}\right) \subseteq S\left(\Delta_{1}\right) \oplus D\left(\Delta_{1}\right) \oplus S\left(\Delta_{2} * \Delta_{3}\right) \oplus D\left(\Delta_{2} * \Delta_{3}\right) .
$$

Definition 2.5.2 Let $\Delta=\Delta_{1} * \Delta_{2} * \Delta_{3} \in \mathcal{B}_{\mathrm{gcd} \neq 0}^{a_{1}}(g)$ and assume that $\operatorname{gcd}_{2}\left(\Delta_{2}, S\left(\Delta_{1}\right), D\left(\Delta_{1}\right)\right)=1$. Let $D\left(\Delta_{1}\right)$ and $D\left(\Delta_{2}\right) \subseteq T\left(\Delta_{1}\right)$ denote a choice of dual summands of $S\left(\Delta_{1}\right)$ and $S\left(\Delta_{2}\right)$, respectively, as in Remark 2.5.1(i). We define $\mathcal{M}_{D\left(\Delta_{1}\right) \mid D\left(\Delta_{2}\right)}^{\Delta_{1}, \Delta_{2}, \Delta_{3}}(g)$ to be the subcomplex of $\mathcal{B}_{\text {gcd }=1 ; t}^{a_{1}, \Delta}(g)$ consisting of simplices $\mathfrak{w}$ which satisfy:

(a) $\operatorname{gcd}_{2}\left(\mathfrak{w}, S\left(\Delta_{1}, \Delta_{2}, \Delta_{3}, D\left(\Delta_{1}\right), D\left(\Delta_{2}\right)\right)\right)=1$.

(b) $\mathfrak{w} \perp D\left(\Delta_{1}\right)$.

The reader should be aware that the role of the first nonempty simplex among $\Delta_{1}, \Delta_{2}, \Delta_{3}$ is to be a bad simplex from Proposition 2.4.7, so we can only assume it is in $\mathcal{B}_{\text {gcd }}^{a_{1}}(g)$. 
Remark 2.5.3 This is the idea: First note that $\mathcal{M}_{0 \mid 0}^{\varnothing, \varnothing, \Delta}(g)=\mathcal{B}_{\text {gcd }=1 ; t}^{a_{1}, \Delta}(g)$, which we need to show is $(g-k-3)$-connected. The following proposition reduces this to showing that $\mathcal{M}_{D(\Delta) \mid 0}^{\Delta, \varnothing, \varnothing}(g)$ is $(g-k-3)$-connected, and in this complex, it is possible to make modifications enough to do division with remainder, as in Proposition 2.5.7: Indeed, if we set $\tilde{f}(s)=\varphi(s)-q_{s} v$, where $v \in f(\sigma)$, then $\operatorname{rk}^{a_{1}}(\tilde{f}(s) \neq 1$. To remedy this, we are forced to use $\widetilde{\varphi}^{\prime}(s)=a_{1}+t b_{1}+\operatorname{pr}_{2}\left(\varphi(s)-q_{s} v\right)$ instead, but then $\widetilde{\varphi}^{\prime}(s)$ is no longer orthogonal to $\Delta$. Here $\mathcal{M}_{D(\Delta) \mid 0}^{\Delta}(g)$ saves the day: All its simplices are orthogonal to $D(\Delta)$, so we can set $\varphi(s)=\widetilde{\varphi}^{\prime}(s)+u_{s}$, where $u_{s} \in D(\Delta)$ satisfies $\varphi(s) \perp \Delta$, without changing anything else $\left(u_{s}\right.$ is constructed in Lemma 2.5.6).

Lemma 2.5.4 Let $\Delta=\Delta_{1} * \Delta_{2} * \Delta_{3} \in \mathcal{B}_{\mathrm{gcd} \neq 0}^{a_{1}}(g)$ be a $k$-simplex. Assume:

- If $\Delta_{1} \neq \varnothing$, that $\Delta_{1} \in \mathcal{B}_{\mathrm{gcd} \neq 0}^{a_{1}}(g), \Delta_{2} \in \mathcal{M}_{0 \mid D\left(\Delta_{1}\right)}^{\varnothing, \Delta_{1}, \varnothing}(g), \Delta_{3} \in \mathcal{M}_{D\left(\Delta_{1}\right) \mid 0}^{\Delta_{1}, \varnothing, \Delta_{2}}(g)$.

- If $\Delta_{1}=\varnothing$, that $\Delta_{2} \in \mathcal{B}_{\mathrm{gcd} \neq 0}^{a_{1}}(g)$ and $\Delta_{3} \in \mathcal{M}_{0 \mid 0}^{\varnothing, \varnothing, \Delta_{2}}(g)=\mathcal{B}_{\mathrm{gcd}=1 ; t}^{a_{1}, \Delta_{2}}(g)$.

- If $\Delta_{1}=\Delta_{2}=\varnothing$, that $\Delta_{3} \in \mathcal{B}_{\text {gcd }}^{a_{1}}(g)$.

Consider the following:

(i) $\pi_{n}\left(\mathcal{M}_{D\left(\Delta_{1}\right) \mid D\left(\Delta_{2}\right)}^{\Delta_{1}, \Delta_{2}, \Delta_{3}}(g)\right)=0$ for $n \leq g-k-3$.

(ii) $\pi_{n}\left(\mathcal{M}_{D\left(\Delta_{1}\right) \mid D\left(\Delta_{2}\right)}^{\Delta_{1}, \Delta_{2}, \varnothing}(g)\right)=0$ for $n \leq g-k-3$.

(iii) $\pi_{n}\left(\mathcal{M}_{D\left(\Delta_{1}\right) \mid 0}^{\Delta_{1}, \varnothing, \varnothing}(g)\right)=0$ for $n \leq g-k-3$.

Then (iii) implies (i) and (ii).

Remark 2.5.5 By the assumptions in the Lemma, one checks that Remark 2.5.1 can be used to create new dual summands, thereby ensuring that

$$
\begin{gathered}
\mathcal{M}_{D\left(\Delta_{1}\right) \mid D\left(\Delta_{2} * \Delta_{3}\right)}^{\Delta_{1}, \Delta_{2} * \Delta_{3}, \varnothing}(g) \subseteq \mathcal{M}_{D\left(\Delta_{1}\right) \mid D\left(\Delta_{2}\right)}^{\Delta_{1}, \Delta_{2}, \Delta_{3}}(g), \\
\mathcal{M}_{D\left(\Delta_{1} * \Delta_{2}\right) \mid 0}^{\Delta_{1} * \Delta_{2}, \varnothing, \varnothing}(g) \subseteq \mathcal{M}_{D\left(\Delta_{1}\right) \mid D\left(\Delta_{2}\right)}^{\Delta_{1}, \Delta_{2}, \varnothing}(g) .
\end{gathered}
$$

Proof We use the same strategy as the proof of Proposition 2.4.7. The argument is inductive in $n$, so let $n$ be fixed.

(i) We inductively assume (i) for all $n^{\prime}<n$, and (ii). Let $f: S \longrightarrow \mathcal{M}_{D\left(\Delta_{1}\right) \mid D\left(\Delta_{2}\right)}^{\Delta_{1}, \Delta_{2}, \Delta_{3}}(g)$ be a simplicial map from a simplicial $n$-sphere $S$. We can construct $D\left(\Delta_{2} * \Delta_{3}\right)$ as in Remark 2.5.1(ii), since $\Delta_{2} * \Delta_{3} \in \mathcal{M}_{0 \mid D\left(\Delta_{1}\right)}^{\varnothing, \Delta_{1}, \varnothing}$.

We say $\sigma \in S$ is regularly bad if for all vertexes $v \in f(\sigma)$, we have

$$
\operatorname{gcd}_{2}\left(v, S\left(f(\sigma) \backslash v, \Delta, D\left(\Delta_{1}\right), D\left(\Delta_{2} * \Delta_{3}\right)\right)\right) \neq 1,
$$


where $f(\sigma) \backslash v$ is the difference between the vertex sets. Let $\sigma$ be a regularly bad simplex of maximal dimension. We claim (see Remark 2.5.5) that

$$
\left.f\right|_{\operatorname{link}(\sigma)}: \operatorname{link}(\sigma) \longrightarrow \mathcal{M}_{D\left(\Delta_{1}\right) \mid D\left(\Delta_{2} * \Delta_{3}\right)}^{\Delta_{1}, \Delta_{2} * \Delta_{3}, f(\sigma)}(g) .
$$

To see this, we must show for all $\tau \subseteq \operatorname{link}(\sigma)$ that

$$
\operatorname{gcd}_{2}\left(f(\tau), S\left(\Delta, f(\sigma), D\left(\Delta_{1}\right), D\left(\Delta_{2} * \Delta_{3}\right)\right)\right)=1 .
$$

The argument is verbatim as in the proof of Proposition 2.4.7(iii) $\Rightarrow$ (ii), replacing $S(f(\sigma))$ by $S\left(\Delta, f(\sigma), D\left(\Delta_{1}\right), D\left(\Delta_{2} * \Delta_{3}\right)\right)$. We can now use (i) inductively to fill put the link in $\mathcal{M}_{D\left(\Delta_{1}\right) \mid D\left(\Delta_{2} * \Delta_{3}\right)}^{\Delta_{1}, \Delta_{2} * \Delta_{3}, f(\sigma)}(g)$, perform a link move to $f$, and check that this creates no new regularly bad simplices, as in the proof of Proposition 2.4.7.

Performing this process inductively, we can assume that there are no regularly bad simplices of $f$. Then by definition, $f(S) \subseteq \mathcal{M}_{D\left(\Delta_{1}\right) \mid D\left(\Delta_{2} * \Delta_{3}\right)}^{\Delta_{1}, \Delta_{2} * \Delta_{3}, \varnothing}(g)$ and by (ii), we are done.

(ii) We say $\sigma \in S$ is regularly bad if all vertices $s$ of $\sigma$ satisfy $s \not \not S\left(\Delta_{2}\right)$. Let $\sigma$ be a regularly bad simplex of maximal dimension. We can choose $D\left(\Delta_{1} * \Delta_{2}\right)=$ $D\left(\Delta_{1}\right) \oplus D\left(\Delta_{2}\right)$ by Remark 2.5.1(i). Now use (i) inductively on $\operatorname{link}(\sigma)$ to fill out the link in $\mathcal{M}_{D\left(\Delta_{1}\right) \oplus D\left(\Delta_{2}\right) \mid 0}^{\Delta_{1} * \Delta_{2}, \varnothing, f(\sigma)}(g)$. After removing all regularly bad simplices, we are in case (iii).

We now construct the $u \in D(\Delta)$ mentioned in Remark 2.5.3:

Lemma 2.5.6 Given $\Delta$, there exists $u \in D(\Delta)$ such that $a_{1}+t b_{1}+u \perp \Delta$.

Proof Write $\Delta=\left(v_{0}, v_{1}, \ldots, v_{k}\right)$. There are two cases:

First if $\operatorname{gcd}_{2}(\Delta)=1$, write $\operatorname{pr}_{2}(\Delta)=\left(v_{0}^{\prime}, \ldots, v_{k}^{\prime}\right)$ (this is a basis of $S(\Delta)$ ). Let $\left(u_{0}, \ldots, u_{k}\right)$ be a dual basis. Set $u=\sum_{j=0}^{k} i_{\text {alg }}\left(v_{j}, a_{1}+t b_{1}\right) u_{j}$. Then $i_{\text {alg }}\left(u, v_{j}^{\prime}\right)=$ $i_{\text {alg }}\left(v_{j}, a_{1}+t b_{1}\right)$. Since $u \in D(\Delta)$, we get for $j=0, \ldots, k$,

$$
i_{\text {alg }}\left(u, v_{j}\right)=i_{\text {alg }}\left(\operatorname{pr}_{2}(u), v_{j}\right)=i_{\text {alg }}\left(u, \operatorname{pr}_{2}\left(v_{j}\right)\right)=i_{\text {alg }}\left(u, v_{j}^{\prime}\right)=-i_{\text {alg }}\left(a_{1}+t b_{1}, v_{j}\right) .
$$

If $\operatorname{gcd}_{2}(\Delta)>1$, then $t=\mathrm{rk}^{b_{1}}\left(v_{0}\right)$; see Proposition 2.4.7(iv). Thus $i_{\text {alg }}\left(a_{1}+t b_{1}, v_{j}\right)=$ $-i_{\text {alg }}\left(\operatorname{pr}_{2}\left(v_{0}\right), v_{j}\right)$. Let $\left(v_{0}^{\prime}, \ldots, v_{k}^{\prime}\right)$ be a basis of $S(\Delta)$, and let $\left(u_{0}, \ldots, u_{k}\right)$ be a dual basis. Set $u=\sum_{j=0}^{k} i_{\text {alg }}\left(\operatorname{pr}_{2}\left(v_{0}\right), v_{j}^{\prime}\right) u_{j}$. Then $i_{\text {alg }}(u, v)=i_{\text {alg }}\left(\operatorname{pr}_{2}\left(v_{0}\right), v\right)$ for $v \in S(\Delta)$. Thus for all $j=0, \ldots, k$,

$$
i_{\text {alg }}\left(u, v_{j}\right)=i_{\text {alg }}\left(u, \operatorname{pr}_{2}\left(v_{j}\right)\right)=i_{\text {alg }}\left(\operatorname{pr}_{2}\left(v_{0}\right), v_{j}\right)=-i_{\text {alg }}\left(a_{1}+t b_{1}, v_{j}\right) .
$$

This shows the lemma. 
Finally we can show the remaining part, (iii) of Lemma 2.5.4. We have given a $k$-simplex $\Delta \in \mathcal{B}_{\mathrm{gcd} \neq 0}^{a_{1}}(g)$, a dual summand $D(\Delta)$, and $T(\Delta)$ as in Remark 2.5.1. To ease the notation, let $\mathcal{N}_{\Delta}(g)=\mathcal{M}_{D(\Delta) \mid 0}^{\Delta, \varnothing, \varnothing}(g)$, and similarly for $\mathfrak{w} \subseteq \mathcal{N}_{\Delta}(g)$, let $\mathcal{N}_{\Delta}^{\mathfrak{w}}(g)=\mathcal{M}_{D(\Delta) \mid 0}^{\Delta, \varnothing, \mathfrak{w}}(g)$ be the link of $\mathfrak{w}$ in $\mathcal{N}_{\Delta}(g)$.

The proof will be similar to the proof of Proposition 2.3.3, and we define: For $x \in T(\Delta)$ with $x \perp \mathfrak{w}$, let $\mathcal{N}_{\Delta}^{\mathfrak{w} ;\langle x\rangle^{\perp}}(g)$ be the subcomplex of $\mathcal{N}_{\Delta}^{\mathfrak{w}}(g)$ consisting of all simplices whose vertices are in $\langle x\rangle^{\perp}$.

Proposition 2.5.7 Let $\Delta \in \mathcal{B}_{\mathrm{gcd} \neq 0}^{a_{1}}(g)$ be a $k$-simplex, and $\mathfrak{w} \in \mathcal{N}_{\Delta}(g)$ an $m$-simplex. Let $x \in T(\Delta)$ with $x \perp \mathfrak{w}$ and $\operatorname{gcd}_{2}(x, \mathfrak{w}, S(\Delta), D(\Delta))=1$. Then:

(i) $\pi_{n}\left(\mathcal{N}_{\Delta}^{\mathfrak{w} ;\langle x\rangle^{\perp}}(g)\right)=0$ for $n \leq g-k-m-4$.

(ii) $\pi_{n}\left(\mathcal{N}_{\Delta}^{\mathfrak{w}}(g)\right)=0$ for $n \leq g-k-m-4$.

Proof We prove (i) inductively, assuming both (i) and (ii) for all $n^{\prime}<n$ and all $k^{\prime}, m^{\prime}$ such that $n^{\prime} \leq g-k^{\prime}-m^{\prime}-4$. We have given a simplicial $n$-sphere $S$ and a simplicial map $f: S \longrightarrow \mathcal{N}_{\Delta}^{\mathfrak{w} ;\langle x\rangle^{\perp}}(g)$. Fix a symplectic basis $\mathfrak{X}$ for $H$ extending $x \in T$ with the dual basis vector $y$ to $x$ also satisfying $y \in T$. Define $R=R_{x}$ as in (9).

If $R=0$, let $u \in D(\Delta)$ with $a_{1}+t b_{1}+u \perp \Delta$ be the element from Lemma 2.5.6. Then proceed as in Proposition 2.3.3, except $F(+)=a_{1}+t b_{1}+x+u$.

If $R>0$ : Call a simplex $\sigma$ in $S$ regularly bad if $\left|\mathrm{rk}^{x}(f(s))\right|=R$ for all vertices $s \in \sigma$. Let $\sigma$ be a regularly bad simplex of maximal dimension. Then

$$
\left.f\right|_{\operatorname{link}(\sigma)}: \operatorname{link}(\sigma) \longrightarrow \mathcal{N}_{\Delta}^{\mathfrak{w} * f(\sigma) ;\langle x\rangle^{\perp}}(g) .
$$

But we cannot be sure $x$ satisfies $\operatorname{gcd}_{2}(x, \mathfrak{w} * f(\sigma), S(\Delta), D(\Delta))=1$. If not, there are two possibilities. To ease the notation write $\mathfrak{w}^{\prime}=\mathfrak{w} * f(\sigma)$.

We will need the following observations time and again: For $h \in H$, let $h_{T}=\operatorname{pr}_{T}(h)$ denote the projection of $h$ on $T$. Then:

(a) $\operatorname{gcd}_{2}(\mathfrak{v}, S(\Delta), D(\Delta))=\operatorname{gcd}_{2}\left(\mathfrak{v}_{T}\right)$.

(b) If $y, v \in D \oplus T$, or if $y \in T, v \in H$, then $i_{\text {alg }}(v, y)=i_{\text {alg }}\left(v_{T}, y\right)$.

The first possibility is $\operatorname{gcd}_{2}\left(x, \mathfrak{w}^{\prime}, S(\Delta), D(\Delta)\right)>1$. Consider the smallest summand $V$ in $T$ containing $\left\langle x, \mathfrak{w}_{T}^{\prime}\right\rangle$. By (b), $V$ is isotropic. By (a), $\operatorname{gcd}_{2}\left(x, \mathfrak{w}_{T}^{\prime}\right)=$ $\operatorname{gcd}_{2}\left(x, \mathfrak{w}^{\prime}, S(\Delta), D(\Delta)\right)>1$, and likewise, $\operatorname{gcd}_{2}\left(\mathfrak{w}_{T}^{\prime}\right)=1$. This means there is a basis of $V$ of the form $\left\{\tilde{x}, \mathfrak{w}_{T}^{\prime}\right\}$. By (a) and (b),

$$
\mathcal{N}_{\Delta}^{\mathfrak{w}^{\prime} ;\langle x\rangle^{\perp}}(g)=\mathcal{N}_{\Delta}^{\mathfrak{w}^{\prime} ;\langle\tilde{x}\rangle^{\perp}}(g) .
$$


One checks that $\tilde{x}$ satisfies all the requirements of the Proposition. So we can use (i) by induction on the map in (24) to obtain $\varphi$ as in (27) below.

The second possibility is $\operatorname{gcd}_{2}\left(x, \mathfrak{w}^{\prime}, S(\Delta), D(\Delta)\right)=0$. Actually,

$$
0=\operatorname{gcd}_{2}\left(x, \mathfrak{w}^{\prime}, S(\Delta), D(\Delta)\right)=\operatorname{gcd}\left(x, \mathfrak{w}_{2}^{\prime}, D(\Delta)\right),
$$

where the second equality uses Remark 2.2.7(ii) along with $\mathfrak{w}^{\prime} \in \mathcal{N}_{\Delta}(g)$. So consider the summand $V=\left\langle\mathfrak{w}_{2}^{\prime}, D(\Delta)\right\rangle$; then $x \in V$. Further, since $\mathfrak{w}^{\prime} \perp D(\Delta)$, we get $\left(\mathfrak{w}_{2}^{\prime}\right)^{i}-\left(\mathfrak{w}_{T}^{\prime}\right)^{i} \in D(\Delta)$ (here, $\mathfrak{v}^{i}$ denotes the $i$-th vertex of $\mathfrak{v}$ ), and thus

$$
V=D(\Delta)+\left\langle\mathfrak{w}_{2}^{\prime}\right\rangle=D(\Delta) \oplus\left\langle\mathfrak{w}_{T}^{\prime}\right\rangle .
$$

So as a basis of $V$, we can take a basis of $D(\Delta)$ along with $x$ and vectors $t_{0}, \ldots, t_{\ell}$, such that $\left\{x, t_{0}, \ldots, t_{\ell}\right\}$ is a basis of $\left\langle\mathfrak{w}_{T}^{\prime}\right\rangle \subseteq T$. We can choose them such that $\operatorname{rk}^{x}\left(t_{j}\right)=0$. Now for $v \in T$, set $\bar{v}=a_{1}+t b_{1}+v+u$, where $u \in D(\Delta)$ is from Lemma 2.5.6 such that $i_{\text {alg }}(\bar{v}, \Delta)=i_{\text {alg }}\left(a_{1}+t b_{1}+u, \Delta\right)=0$. We consider $\left(\bar{x}, \bar{t}_{0}, \ldots, \bar{t}_{\ell}\right)$, which is isotropic, since $V$ is easily shown to be isotropic. In fact it is a simplex in $\mathcal{N}_{\Delta}(g)$, since

$$
\begin{aligned}
1 & =\operatorname{gcd}_{2}\left(\mathfrak{w}^{\prime}, S(\Delta), D(\Delta)\right)=\operatorname{gcd}_{2}\left(\mathfrak{w}_{T}^{\prime}, S(\Delta), D(\Delta)\right) \\
& =\operatorname{gcd}_{2}\left(x, t_{0}, \ldots, t_{\ell}, S(\Delta), D(\Delta)\right)=\operatorname{gcd}_{2}\left(\bar{x}, \bar{t}_{0}, \ldots, \bar{t}_{\ell}, S(\Delta), D(\Delta)\right) .
\end{aligned}
$$

The last equality holds since $u \in D(\Delta)$. A slight modification of (26) along with (a) and (b) shows $\mathcal{N}_{\Delta}^{\mathfrak{w}^{\prime} ;\langle x\rangle^{\perp}}(g)=\mathcal{N}_{\Delta}^{\left(\bar{x}, \bar{t}_{0}, \ldots, \bar{t}_{\ell}\right) ;\langle x\rangle^{\perp}}(g)$. Completely analogously to the proof of Proposition 2.3.3 (see (15)), we can then factor $\left.f\right|_{\operatorname{link}(\sigma)}$ as

$$
\left.f\right|_{\operatorname{link}(\sigma)}: \operatorname{link}(\sigma) \subseteq B \stackrel{\varphi}{\longrightarrow} \mathcal{N}_{\Delta}^{\mathfrak{w} * f(\sigma) ;\langle x\rangle^{\perp}}(g) .
$$

We now modify $\varphi$ to a map $\varphi^{\prime}$ by performing division with remainder: Let $v=f(t)$ for some fixed vertex $t \in \sigma$, and write $v_{s}=\varphi(s)$ for $s \in B^{(0)}$. By division we obtain $q_{s}$ such that $\left|\mathrm{rk}^{x}\left(v_{s}-q_{s} v\right)\right|<\left|\mathrm{rk}^{x}(v)\right|=R$ for all $s \in B^{(0)}$. For $s \in \partial B=\operatorname{link}(\sigma)$ we take $q_{s}=0$ so we do not change $\varphi$ on $\operatorname{link}(\sigma)$. Let $u \in D(\Delta)$ be the vector from Lemma 2.5.6 such that $a_{1}+t b_{1}+u \perp \Delta$. We then set (cf Remark 2.5.3)

$$
\begin{aligned}
\varphi^{\prime}(s) & =v_{s}-q_{s} v+q_{s}\left(a_{1}+t b_{1}+u\right) \\
& =a_{1}+t b_{1}+\operatorname{pr}_{2}\left(\varphi(s)-q_{s} f(t)\right)+q_{s} u,
\end{aligned}
$$

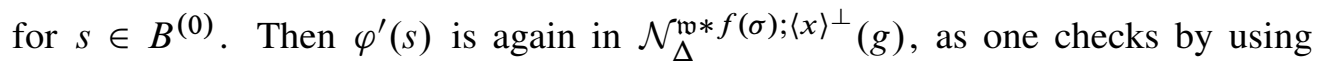
$u \in D(\Delta), D(\Delta)$ is isotropic and $x \in T$. Then $\operatorname{rk}^{x}\left(\varphi^{\prime}(s)\right)=i_{\text {alg }}\left(\varphi^{\prime}(s), y\right)=$ $i_{\text {alg }}\left(v_{s}-q_{s} v, y\right)$, since $y$ is the dual basis vector to $x$, and $y \in T$, so $y \perp u$. The result is thus a simplicial map $\varphi^{\prime}: B \longrightarrow \mathcal{N}_{\Delta}^{\mathfrak{w} * f(\sigma) ;\langle x\rangle^{\perp}}(g)$ with $\left|\operatorname{rk}^{x}\left(\varphi^{\prime}(s)\right)\right|<R$ for 
all $s \in B^{(0)}$. Then we do a link move to $f$ on $\sigma$ with $\varphi^{\prime}$, which produces a map homotopic to $f$, removing $\sigma$. Iterating this, we obtain $R=0$.

We conclude (ii) from (i) precisely as in the proof of Proposition 2.3.3(ii).

Corollary 2.5.8 $\mathcal{B}_{\mathrm{gcd}=1 ; t}^{a_{1}, \Delta^{k}}(g)$ is $(g-k-3)$-connected.

Proof Choose a dual summand $D=D\left(\Delta^{k}\right)$ to $S\left(\operatorname{pr}_{2}\left(\Delta^{k}\right)\right)$. By Proposition 2.5.7, $N_{\Delta^{k}}(g)=\mathcal{M}_{D \mid 0}^{\Delta^{k}, \varnothing, \varnothing}(g)$ is $(g-k-3)$-connected. By Proposition 2.4.7 (iii) $\Rightarrow$ (i), we then obtain that $\mathcal{M}_{0 \mid 0}^{\varnothing, \varnothing, \Delta^{k}}(g)=\mathcal{B}_{\text {gcd }=1 ; t}^{a_{1}, \Delta^{k}}(g)$ is $(g-k-3)$-connected.

\section{Exactness in the spectral sequence}

Let $H(m)=H_{1}\left(F_{m, 1} ; \mathbb{Z}\right)$ with given symplectic basis $\left(a_{1}, b_{1}, \ldots, a_{m}, b_{m}\right)$. Let $i=1,2$, put $H=H(g+i-1)$, and $H_{\mathbb{Q}}=H \otimes \mathbb{Q}$. Always assume $g \geq 6$.

In this section, we finish the proof of Theorem 1.0.1. Recall we must show

$$
E_{2,1}^{2}\left(F_{g, 1} ; 1\right)=0, \quad E_{2,1}^{2}\left(F_{g-1,2} ; 2\right)=0 .
$$

Write $E_{2,1}^{1}=E_{2,1}^{1}\left(F_{g, i} ; i\right)$. That $E_{2,1}^{2}=0$ is equivalent to the sequence $E_{1,1}^{1} \longleftarrow$ $E_{2,1}^{1} \longleftarrow E_{3,1}^{1}$ being exact.

To show this, we need a more concrete description of the spectral sequence for $q=1$. We shall use the following result of [10, Theorem 3.5.6]. See also [6, Theorem 1.2].

Theorem 3.0.1 [10] Let $S$ be a subsurface of $F_{g, 1}$, obtained from $F_{g, 1}$ by cutting along arcs $\gamma_{1}, \ldots, \gamma_{n}$, where $\left(\gamma_{1}, \ldots, \gamma_{n}\right) \in C_{*}\left(F_{g, 1}, 1\right)$. Let $\tau_{F_{g, 1}, S}$ denote the restriction to $\mathcal{I}\left(F_{g, 1}, S\right)$ of the Johnson homomorphism $\tau_{g, 1}: \mathcal{I}_{g, 1} \longrightarrow \bigwedge^{3} H$. Let $c_{j}$ be the homology class of $\tilde{\gamma}_{j}$ (see Figure 4) in $H_{\mathbb{Q}}$. Assume the genus of $S$ is at least 3. Then

$$
H_{1}\left(\mathcal{I}\left(F_{g, 1}, S\right) ; \mathbb{Q}\right) \cong \operatorname{im}\left(\tau_{F_{g, 1}, S}\right) \otimes \mathbb{Q} \cong \bigwedge^{3}\left\langle c_{1}, \ldots, c_{n}\right\rangle_{\mathbb{Q}}^{\perp} \subseteq \bigwedge^{3} H_{\mathbb{Q}} .
$$

Using this we get from (6),

$$
E_{p, 1}^{1}\left(F_{g, 1} ; 1\right) \cong \bigoplus_{\mathfrak{w} \in \mathcal{B}(g)^{(p-1)}} \bigwedge^{3}\langle\mathfrak{w}\rangle_{\mathbb{Q}}^{\perp} \Rightarrow 0 \quad \text { for } p+1 \leq g-1 .
$$

Likewise from (7), using that $F_{g, 2}=\left(F_{g+1,1}\right)_{\beta}$, where $\widetilde{\beta} \simeq \beta_{1}$ (see Figure 1),

$$
E_{p, 1}^{1}\left(F_{g, 2} ; 2\right) \cong \bigoplus_{\mathfrak{w} \in \mathcal{B}^{a_{1}}(g)^{(p-1)}} \bigwedge^{3}\left\langle\mathfrak{w}, b_{1}\right\rangle_{\mathbb{Q}}^{\perp} \Rightarrow 0 \quad \text { for } p+1 \leq g .
$$


To enable us to talk about both cases simultaneously, define

$$
b^{i}= \begin{cases}\varnothing & i=1, \\ b_{1} & i=2 .\end{cases}
$$

The differentials $d_{p, 1}^{1}$ have the following description under the isomorphisms (28) and (29) above: Let $\partial_{j}$ denote the $j$-th face map in $\mathcal{B}(g)$, ie if $\mathfrak{w}=\left(w_{0}, \ldots, w_{p}\right)$ then $\partial_{j} \mathfrak{w}=\left(w_{0}, \ldots, \widehat{w}_{j}, \ldots, w_{p}\right)$. Write an element of $E_{p, 1}^{i}$ as $(v, \mathfrak{w})$ where $v \in$ $\bigwedge^{3}\left\langle\mathfrak{w}, b^{i}\right\rangle_{\mathbb{Q}}^{\perp}$. Then $d_{p, 1}^{1}$ is the linear map given by

$$
d_{p, 1}^{1}(v, \mathfrak{w})=\sum_{j=0}^{p-1}(-1)^{j}\left(i_{j}(v), \partial_{j} \mathfrak{w}\right),
$$

where $i_{j}: \bigwedge^{3}\left\langle\mathfrak{w}, b^{i}\right\rangle_{\mathbb{Q}}^{\perp} \longrightarrow \bigwedge^{3}\left\langle\partial_{j} \mathfrak{w}, b^{i}\right\rangle_{\mathbb{Q}}^{\perp}$ denotes the inclusion.

\subsection{Discrete Morse vector field basics}

We shall use the an adaptation of the technique of discrete Morse theory on a chain complex $E_{*}$. The difference from ordinary discrete Morse theory is that we cannot define a gradient vector field directly on the simplices, but rather have to work in the chain groups $E_{n}$. The approach actually resembles collapsing schemes more than discrete Morse theory, but we assume more people are familiar with the discrete Morse theory approach, so we use that terminology. The following example hopefully clarifies the ideas:

Example 3.1.1 (Discrete Morse vector field) To give the idea and motivate the definition, we consider an example, namely the full subcomplex of $\mathcal{B}(g)$ spanned by the four vertices, $a_{1}, a_{2}, a_{3}, a_{4} \in H$. The corresponding chain groups are then (see Section 3.2 for a technical detail here):

$$
\begin{aligned}
& E_{0}=\bigwedge^{3} H, \\
& E_{1}=\bigwedge^{3}\left\langle a_{1}\right\rangle_{\mathbb{Q}}^{\perp} \oplus \bigwedge^{3}\left\langle a_{2}\right\rangle_{\mathbb{Q}}^{\perp} \oplus \bigwedge^{3}\left\langle a_{3}\right\rangle_{\mathbb{Q}}^{\perp} \oplus \bigwedge^{3}\left\langle a_{4}\right\rangle_{\mathbb{Q}}^{\perp}, \\
& E_{2}=\bigoplus_{i=1}^{4} \bigoplus_{j=1}^{i-1} \bigwedge^{3}\left\langle a_{i}, a_{j}\right\rangle_{\mathbb{Q}}^{\perp},
\end{aligned}
$$

with the differential described in (31). To show the sequence $E_{0} \leftarrow E_{1} \leftarrow E_{2}$ is exact, we argue as follows. First, we decompose

$$
E_{0}=\bigwedge^{3} H \cong \bigwedge^{3}\left\langle a_{1}\right\rangle_{\mathbb{Q}}^{\perp} \oplus\left\langle b_{1}\right\rangle \wedge \bigwedge^{2}\left\langle a_{2}\right\rangle_{\mathbb{Q}}^{\perp} \oplus\left\langle b_{1} \wedge b_{2}\right\rangle \wedge\left\langle a_{3}\right\rangle_{\mathbb{Q}}^{\perp} \oplus\left\langle b_{1} \wedge b_{2} \wedge b_{3}\right\rangle .
$$

We see that $d: E_{1} \longrightarrow E_{0}$ is surjective, and furthermore is injective from the summand $\mathcal{C}_{1} \subseteq E_{1}$ defined by the right-hand side of (32). If we choose bases for each of the 
summands, we can pair each basis vector $x^{(0)}$ of $E_{0}$ with the corresponding basis vector $y^{(1)}$ of $\mathcal{C}_{1}$, such that $d\left(y^{(1)}\right)=x^{(0)}$. By corresponding, we mean: For each simplex $\mathfrak{v}$ there is an inclusion $i_{\mathfrak{v}}: \bigwedge^{3}\langle\mathfrak{v}\rangle_{\mathbb{Q}}^{\perp} \longrightarrow \bigwedge^{3} H$, and two vectors correspond if their images under the respective inclusions agree. These pairs $\left(x^{(0)}, y^{(1)}\right)$ make up (part of) a discrete Morse vector field, which will be defined below. We now intend to pair the thus far unpaired basis vectors of $E_{1}$ with basis vectors of $E_{2}$, in a similar manner. We have $E_{1}=\mathcal{C}_{1} \oplus \mathcal{R}_{1}$, where

$$
\begin{aligned}
\mathcal{R}_{1}=0 \oplus \bigwedge^{3}\left\langle a_{1}, a_{2}\right\rangle_{\mathbb{Q}}^{\perp} \oplus\left(\bigwedge^{3}\left\langle a_{1}, a_{3}\right\rangle \frac{\perp}{\mathbb{Q}} \oplus\left\langle b_{1}\right\rangle \wedge \bigwedge^{2}\left\langle a_{2}\right\rangle_{\mathbb{Q}}^{\perp}\right) \\
\oplus\left(\bigwedge^{3}\left\langle a_{1}, a_{4}\right\rangle_{\mathbb{Q}}^{\perp} \oplus\left\langle b_{1}\right\rangle \wedge \bigwedge^{2}\left\langle a_{2}, a_{4}\right\rangle_{\mathbb{Q}}^{\perp} \oplus\left\langle b_{1} \wedge b_{2}\right\rangle \wedge\left\langle a_{3}, a_{4}\right\rangle_{\mathbb{Q}}^{\perp}\right)
\end{aligned}
$$

Again, (33) also defines a summand of $E_{2}$, which we call $\mathcal{C}_{2}$, and we pair each basis vector $x^{(1)}$ of $\mathcal{R}_{1}$ with the corresponding vector $y^{(2)}$ in $\mathcal{C}_{2}$. This time, we have $d\left(y^{(2)}\right)=x^{(1)}+z^{(1)}$, where $z^{(1)} \in C_{1}$. This still suffices to show $E_{2} \rightarrow E_{1} \rightarrow E_{0}$ is exact, as can be verified directly, or seen from Proposition 3.1.5 below.

With this example in mind, we make the general definition

Definition 3.1.2 (Discrete vector field) Given a chain complex $E_{*}$ with chain groups $E_{n}$ and differential $d$, and for each $n$ a choice of basis $\operatorname{Bas}_{n}$ of $E_{n}$, then a discrete vector field $V_{*}=\left\{V_{n}\right\}_{n \geq 0}$ on $E_{*}$ is for each $n$ a collection $V_{n}$ of basis vector pairs, $\left(x^{(n)}, y^{(n+1)}\right)$, where $x^{(n)} \in \mathrm{Bas}_{n}$ and $y^{(n+1)} \in \mathrm{Bas}_{n+1}$ satisfying (i) and (ii):

(i) (Compatibility with $d$ ) For each pair $\left(x^{(n)}, y^{(n+1)}\right) \in V_{n}$,

$$
d\left(y^{(n+1)}\right)=x^{(n)}+\sum_{z \in \mathrm{Bas}_{n}, z \neq x} n_{z} \cdot z,
$$

where each $n_{z} \in \mathbb{Q}$.

(ii) (Disjoint pairs) Each basis vector in $\bigcup_{n} \operatorname{Bas}_{n}$ belongs to at most 1 pair of $V_{*}$.

The construction in Example 3.1.1 defines a discrete vector field.

Definition 3.1.3 (Morse vector field) A gradient path (at level $n$ ) for $V_{*}$ is a sequence

$$
\left(x_{0}^{(n)}, y_{0}^{(n+1)}\right) \rightarrow\left(x_{1}^{(n)}, y_{1}^{(n+1)}\right) \rightarrow\left(x_{2}^{(n)}, y_{2}^{(n+1)}\right) \rightarrow \cdots,
$$

with $\left(x_{j}, y_{j}\right) \in V_{n}$, and for each $j, x_{j+1}$ has a nonzero coefficient in the basis expansion of $d\left(y_{j}\right)$, but $x_{j+1} \neq x_{j}$. If $V_{*}$ has no infinite gradient paths (in particular no loops), we call it a Morse vector field. 
The discrete vector field from Example 3.1.1 is a Morse vector field, since each gradient path at level 0 has length 0, and at level 1 has length at most 3. (Starting with a basis vector in $\bigwedge^{3}\left\langle a_{4}\right\rangle_{\mathbb{Q}}^{\perp} \subseteq E_{1}$.)

Definition 3.1.4 Define the space of redundant vectors,

$$
\mathcal{R}_{n}=\operatorname{span}\left\{x^{(n)} \mid \exists y^{(n+1)} \in \operatorname{Bas}_{n+1}:(x, y) \in V_{n}\right\}
$$

and the space of collapsible vectors

$$
\mathcal{C}_{n}=\operatorname{span}\left\{y^{(n)} \mid \exists x^{(n-1)} \in \operatorname{Bas}_{n-1}:(x, y) \in V_{n-1}\right\} .
$$

Note, due to the disjoint pairs requirement (Definition 3.1.2(ii)), $\mathcal{R}_{n}$ and $\mathcal{C}_{n}$ form a direct sum in $E_{n}$.

For a subspace $A \subseteq E_{n}$, we say $V_{*}$ spans $A$, if $A \subseteq \mathcal{R}_{n} \oplus \mathcal{C}_{n}$.

The goal is to construct a Morse vector field that spans $E_{0}^{i}, E_{1}^{i}$, and $E_{2}^{i}$. Then $E_{0}^{i} \longleftarrow E_{1}^{i} \longleftarrow E_{2}^{i}$ is exact, as the following Proposition shows:

Proposition 3.1.5 Given a Morse vector field $V_{*}$ on a chain complex $E_{*}$.

(i) The restricted differential map $\left.d\right|_{C_{n}}: C_{n} \longrightarrow E_{n-1}$ is injective.

(ii) If $V_{*}$ spans $E_{n}$, then the chain complex is exact at $E_{n}$.

Proof sketch Both these properties come from the fact that there are no infinite gradient paths, and in particular no loops. This gives (i), since it means contributions from different $d(y)$ 's cannot cancel each other out completely (since otherwise, there would be a loop). To prove (ii), given $v \in E_{n}$ with $d(v)=0$, the same argument works to reduce $v$ (in a finite number of steps) to a collapsible vector modulo the image of $d$. Apply (i).

\subsection{Reducing to a simplicial complex}

This section deals with a technical point: Both $\mathcal{B}(g)$ and $\mathcal{B}^{a_{1}}(g)$ are multisimplicial complexes. This means in the description of $E_{p, 1}^{1}$ from (28) and (29) there occur multiple copies of the same summand $\bigwedge^{3}\langle\mathfrak{w}\rangle_{\mathbb{Q}}^{\perp}$, since the order of the vectors is irrelevant.

To avoid this redundancy, we introduce simplicial complexes, as follows:

Given a multisimplicial complex $\mathcal{B}$, and a total ordering $\mathcal{O}$ on the vertices of $\mathcal{B}$, define $\mathcal{O B}$ to be the subcomplex of $\mathcal{B}$ consisting of simplices with vertices in ascending order. Then $\mathcal{O B}$ is a simplicial complex. 
We will first construct a Morse vector field for the simplicial complexes $\mathcal{B}^{1}(g)=\mathcal{O B}(g)$ and $\mathcal{B}^{2}(g)=\mathcal{O B}^{a_{1}}(g)$. We write a simplex in $\mathcal{B}^{i}(g)$ as a set of vectors $\left\{v_{0}, \ldots, v_{n}\right\}$, which is unambiguous since the order is fixed, to distinguish it from a simplex in $\mathcal{B}(g)$ or $\mathcal{B}^{a_{1}}(g)$.

Definition 3.2.1 Let $i \in\{1,2\}$. Define $E_{*}^{i}$ to be the chain complex with chain groups $E_{n}^{i}=\bigoplus_{\mathfrak{w} \in \mathcal{B}^{i}(g)} \wedge^{3}\left\langle\mathfrak{w}, b^{i}\right\rangle_{\mathbb{Q}}^{\perp}$, and differential as in (31).

Remark 3.2.2 The proofs from Section 2, Propositions 2.3.1 and 2.4.1, work verbatim to give that $\mathcal{B}^{1}(g)$ and $\mathcal{B}^{2}(g)$ are $(g-2)$-connected.

The main result regarding the chain complex $E_{*}^{i}$ is:

Theorem 3.2.3 Assume $g \geq 8-i$, and let $i \in\{1,2\}$. There is a Morse vector field on $E_{*}^{i}$ that spans $E_{0}^{i}, E_{1}^{i}$, and $E_{2}^{i}$.

Proving this will the aim of the next sections. We first note that Theorem 3.2.3 can be extended to $\mathcal{B}(g)$ and $\mathcal{B}^{a_{1}}(g)$ :

Proposition 3.2.4 Let $i \in\{1,2\}$ and $g \geq 8-i$. Assume that the Morse vector field on $E_{*}^{i}$ constructed in Section 3.4 spans $E_{0}^{i}, E_{1}^{i}$, and $E_{2}^{i}$. Then there exists a Morse vector field on $E_{*, 1}^{1}$ spanning $E_{0,1}^{1}, E_{1,1}^{1}$ and $E_{2,1}^{1}$.

Proof The proof relies on details of the construction, and is therefore relegated to the end of Section 3.4.

As a corollary, by Proposition 3.1.5 we have: For $g \geq 7, E_{2,1}^{2}\left(F_{g, 1} ; 1\right)=0$ and $E_{2,1}^{2}\left(F_{g-1,2} ; 2\right)=0$. This finishes the proof of the main result, Theorem 1.0.1.

Consequently, all that remains is to prove Theorem 3.2.3.

\subsection{Morse vector field construction strategy}

In this and the following sections, we prove Theorem 3.2.3. This section contains the main elements of the construction, while the next Section 3.4 actually pieces the vector field together. Finally, the last two Section 3.5 and Section 3.6 verify that is indeed a Morse vector field for $i=1$ and $i=2$, respectively.

We briefly recall the setup:

$$
E_{n}^{i}=\bigoplus_{\mathfrak{w} \in \mathcal{B}^{i}(g)} \bigwedge^{3}\left\langle\mathfrak{w}, b^{i}\right\rangle_{\mathbb{Q}}^{\perp}
$$


where

$$
b^{i}= \begin{cases}\varnothing & i=1, \\ b_{1} & i=2,\end{cases}
$$

and $\mathcal{B}^{i}(g)$ is the simplicial complex obtained from the multisimplicial complex of ordered symplectic bases $\mathcal{B}(g)$ for $i=1$ and from $\mathcal{B}^{a_{1}}(g)$ for $i=2$, respectively, by choosing a total ordering, as explained in Section 3.2 above.

The goal is to choose bases of $E_{n}^{i}$ for $n=0,1,2,3$ and construct a discrete Morse vector field spanning $E_{1}^{i}$ and $E_{2}^{i}$. This will mean exactness at $E_{2}^{i}$, as desired.

The construction is simplified by the following observation, which was also used implicitly in Example 3.1.1:

Remark 3.3.1 Given a choice of basis $B_{n}=\operatorname{Bas}_{n}$ of $\mathcal{R}_{n}$, each basis vector $x \in B_{n}$ is in some $\bigwedge^{3}\left\langle\mathfrak{w}, b^{i}\right\rangle \stackrel{\perp}{\mathbb{Q}}$ for some simplex $\mathfrak{w} \in \mathcal{B}^{i}(g)^{(n-1)}$. We write $\mathfrak{w}=\operatorname{simp}(z)$. We must specify the basis vector $y=c(x)$ paired with $x$. Then by (i) in Definition 3.1.3, we must have $\operatorname{simp}(c(x))=\mathfrak{v} \in \mathcal{B}^{i}(g)^{(n)}$, where $\mathfrak{w}$ is a face of $\mathfrak{v}$. The are natural inclusions $i_{\mathfrak{w}}: \bigwedge^{3}\langle\mathfrak{w}\rangle \stackrel{\perp}{\mathbb{Q}} \longrightarrow \bigwedge^{3} H_{\mathbb{Q}}$ for each $\mathfrak{w}$. Then $c(x) \in E_{n+1}^{i}$ is specified uniquely by requiring that $i_{\mathfrak{w}}(x)=i_{\mathfrak{v}}(c(x))$. So to define the vector field $V_{n}$, we need only specify the basis $B_{n}$ of $\mathcal{R}_{n}$, and for each basis vector $x \in B_{n}$ choose the simplex $\operatorname{simp}(c(x)) \in \mathcal{B}^{i}(g)^{(n+1)}$.

Basically, the construction uses the decomposition idea of Example 3.1.1. We need dual vectors, as in Proposition 2.2.9.

Lemma 3.3.2 Let $i \in\{1,2\}$. Given a simplex $\mathfrak{w} \in \mathcal{B}^{i}(g)$, assume there exists $\left\{w_{1}, w_{2}, w_{3}, w_{4}\right\} \in \mathcal{B}^{i}(g)$ with $\mathfrak{w} *\left\{w_{1}, w_{2}, w_{3}, w_{4}\right\} \in \mathcal{B}^{i}(g)$. Let $u_{j}$ be a dual vector to $w_{j}$ with $i_{\text {alg }}\left(w, u_{j}\right)=0$ for each vertex $w \in \mathfrak{w}$ and each $j=1,2,3,4$. Then there is an isomorphism

$$
\begin{aligned}
\bigwedge^{3}\left\langle\mathfrak{w}, b^{i}\right\rangle_{\mathbb{Q}}^{\perp} \cong \bigwedge^{3}\left\langle\mathfrak{w}, w_{1}, b^{i}\right\rangle_{\mathbb{Q}}^{\perp} \oplus\left\langle u_{1}\right\rangle \wedge \wedge^{2}\left\langle\mathfrak{w}, w_{2}, b^{i}\right\rangle_{\mathbb{Q}}^{\perp} \\
\oplus\left\langle u_{1} \wedge u_{2}\right\rangle \wedge\left\langle\mathfrak{w}, w_{3}, b^{i}\right\rangle_{\mathbb{Q}}^{\perp} \oplus\left\langle u_{1} \wedge u_{2} \wedge u_{3}\right\rangle .
\end{aligned}
$$

We call this the decomposition of $\bigwedge^{3}\left\langle\mathfrak{w}, b^{i}\right\rangle_{\mathbb{Q}}^{\perp}$ with respect to $w_{1}, w_{2}, w_{3}, w_{4}$. (Note it also depends on a choice of dual vectors.)

Using Lemma 3.3.2, we can define a vector field on $\bigwedge^{3}\left\langle\mathfrak{w}, b^{i}\right\rangle_{\mathbb{Q}}^{\perp}$ as follows:

Corollary 3.3.3 Given $\mathfrak{w} \in \mathcal{B}^{i}(g)$. Assume there are $w_{1}, w_{2}, w_{3}, w_{4}$ with $\mathfrak{w} *$ $\left\{w_{1}, w_{2}, w_{3}, w_{4}\right\} \in \mathcal{B}^{i}(g)$. Then there is a vector field that spans $\bigwedge^{3}\left\langle\mathfrak{w}, b^{i}\right\rangle \stackrel{\perp}{\mathbb{Q}}$. 
Proof Write $\mathfrak{w}=\left\{v_{1}, \ldots, v_{n}\right\}$. Choose dual vectors $y_{1}, \ldots, y_{n}, u_{1}, \ldots, u_{4}$ to $v_{1}, \ldots, v_{n}, w_{1}, \ldots, w_{4}$; then we can use Lemma 3.3 .2 to decompose $\bigwedge^{3}\left\langle\mathfrak{w}, b^{i}\right\rangle \frac{\perp}{\mathbb{Q}}$. Choose a basis $B_{w_{i}}$ of each of the four summands in the decomposition. For $z \in B_{w_{i}}$, set $\operatorname{simp}(c(z))=\mathfrak{w} *\left\{w_{i}\right\}$. Then set $B_{w}=B_{w_{1}} \cup B_{w_{2}} \cup B_{w_{3}} \cup B_{w_{4}}$.

After having done as in Corollary 3.3.3 above, we have defined some collapsible vectors inside $\bigoplus_{j=1}^{4} \wedge^{3}\left\langle\mathfrak{w}, w_{j}\right\rangle_{\mathbb{Q}}$. We will need to make the rest of these four summands redundant:

Lemma 3.3.4 Given $\mathfrak{w} \in \mathcal{B}^{i}(g)$. Assume there are vectors $w_{1}, w_{2}, w_{3}, w_{4}$ satisfying $\mathfrak{w} *\left\{w_{1}, w_{2}, w_{3}, w_{4}\right\} \in \mathcal{B}^{i}(g)$. Let $C\left(w_{j}\right)$ be the summands of Lemma 3.3.2:

$$
\begin{aligned}
& \mathcal{C}\left(w_{1}\right)=\wedge^{3}\left\langle\mathfrak{w}, w_{1}, b^{i}\right\rangle_{\mathbb{Q}}^{\perp}, \quad \mathcal{C}\left(w_{2}\right)=\left\langle u_{1}\right\rangle \wedge \bigwedge^{2}\left\langle\mathfrak{w}, w_{2}, b^{i}\right\rangle_{\mathbb{Q}}^{\perp}, \\
& \mathcal{C}\left(w_{3}\right)=\left\langle u_{1} \wedge u_{2}\right\rangle \wedge\left\langle\mathfrak{w}, w_{3}, b^{i}\right\rangle_{\mathbb{Q}}^{\perp}, \quad \mathcal{C}\left(w_{4}\right)=\left\langle u_{1} \wedge u_{2} \wedge u_{3}\right\rangle .
\end{aligned}
$$

Define $\mathcal{R}\left(w_{1}\right)=0, \mathcal{R}\left(w_{2}\right)=\bigwedge^{3}\left\langle\mathfrak{w}, w_{1}, w_{2}, b^{i}\right\rangle_{\mathbb{Q}}^{\perp}$, and

$$
\begin{aligned}
\mathcal{R}\left(w_{3}\right)= & \bigwedge^{3}\left\langle\mathfrak{w}, w_{1}, w_{3}, b^{i}\right\rangle_{\mathbb{Q}}^{\perp} \oplus\left\langle u_{1}\right\rangle \wedge \bigwedge^{2}\left\langle\mathfrak{w}, w_{2}, w_{3}, b^{i}\right\rangle_{\mathbb{Q}}^{\perp}, \\
\mathcal{R}\left(w_{4}\right)=\bigwedge^{3}\left\langle\mathfrak{w}, w_{1}, w_{4}, b^{i}\right\rangle_{\mathbb{Q}}^{\perp} \oplus\left\langle u_{1}\right\rangle \wedge & \bigwedge^{2}\left\langle\mathfrak{w}, w_{2}, w_{4}, b^{i}\right\rangle_{\mathbb{Q}}^{\perp} \\
& \oplus\left\langle u_{1} \wedge u_{2}\right\rangle \wedge\left\langle\mathfrak{w}, w_{3}, w_{4}, b^{i}\right\rangle_{\mathbb{Q}}^{\perp}
\end{aligned}
$$

Then, $\bigwedge^{3}\left\langle\mathfrak{w}, w_{j}\right\rangle_{\mathbb{Q}}^{\perp}=\mathcal{C}\left(w_{j}\right) \oplus \mathcal{R}\left(w_{j}\right)$.

Corollary 3.3.5 Given $\mathfrak{w} \in \mathcal{B}^{i}(g)$. Assume there are vectors $w_{1}, w_{2}, w_{3}, w_{4}$ satisfying $\mathfrak{w} *\left\{w_{1}, w_{2}, w_{3}, w_{4}\right\} \in \mathcal{B}^{i}(g)$. Then there is a vector field on $E_{*}^{i}$ that spans $\bigoplus_{j=1}^{4} \bigwedge^{3}\left\langle\mathfrak{w}, w_{j}, b^{i}\right\rangle_{\mathbb{Q}}^{\perp}$

Proof From Corollary 3.3.3 we have chosen $\bigoplus_{j=1}^{4} \mathcal{C}\left(w_{j}\right)$ to be collapsible inside $\bigoplus_{j=1}^{4} \bigwedge^{3}\left\langle\mathfrak{w}, w_{j}\right\rangle_{\mathbb{Q}}^{\perp}$, in the notation of Lemma 3.3.4. This Lemma also gives a decomposition of the rest, allowing us to choose bases $B\left(w_{j}\right)$ for $R\left(w_{j}\right)$ of the form $B\left(w_{j}\right)=$ $B_{1}\left(w_{j}\right) \cup \cdots \cup B_{j-1}\left(w_{j}\right)$. Here $B_{1}\left(w_{j}\right)$ is a basis of $\bigwedge^{3}\left\langle\mathfrak{w}, w_{1}, w_{j}\right\rangle_{\mathbb{Q}}^{\perp}, B_{2}\left(w_{j}\right)$ is a basis of $\left\langle u_{1}\right\rangle \wedge \wedge^{2}\left\langle\mathfrak{w}, w_{2}, w_{j}\right\rangle_{\mathbb{Q}}^{\perp}$, and $B_{3}\left(w_{4}\right)$ is a basis of $\left\langle u_{1} \wedge u_{2}\right\rangle \wedge\left\langle\mathfrak{w}, w_{3}, w_{4}\right\rangle_{\mathbb{Q}}$. For each basis vector $b \in B_{m}\left(w_{j}\right)$ we assign $\operatorname{simp}(c(b))=\mathfrak{w} *\left\{w_{m}, w_{j}\right\}$.

The difficulty will be ensuring there are no infinite gradient paths for the constructed vector field. The idea is to define filtrations

- $\mathcal{F}_{\infty}^{1} \subset \mathcal{F}_{5}^{1} \subset \mathcal{F}_{4}^{1} \subset \mathcal{F}_{3}^{1} \subset \mathcal{F}_{2}^{1} \subset \mathcal{F}_{1}^{1}=\mathcal{B}^{1}(g)$ on $\mathcal{B}^{1}(g)$,

- $\mathcal{F}_{\infty}^{2} \subset \mathcal{F}_{5}^{2} \subset \mathcal{F}_{4}^{2} \subset \mathcal{F}_{3}^{2} \subset \mathcal{F}_{2}^{2} \subset \mathcal{F}_{1}^{2} \subset \mathcal{F}_{0}^{2}=\mathcal{B}^{2}(g)$ on $\mathcal{B}^{2}(g)$. 
Then for each simplex $\mathfrak{w}$, we will choose $w_{1}, w_{2}, w_{3}, w_{4}$ as in Corollary 3.3.5 in a lower filtration than $\mathfrak{w}$, allowing us to argue inductively. We introduce the notation

$$
A_{1}=a_{1}, \quad A_{j}=\left\{\begin{array}{ll}
a_{j} & \text { in } \mathcal{B}^{1}(g), \\
a_{1}+a_{j} & \text { in } \mathcal{B}^{2}(g),
\end{array} \text { for } j=2,3,4 .\right.
$$

We choose fixed dual vectors to the vertices $A_{1}, A_{2}, A_{3}, A_{4}$ as follows:

$$
B_{1}=\left\{\begin{array}{ll}
b_{1} & \text { for } \mathcal{B}^{1}(g), \\
b_{1}-b_{2}-b_{3}-b_{4} & \text { for } \mathcal{B}^{2}(g),
\end{array} \quad B_{j}=b_{j} \text { for } j=2,3,4 .\right.
$$

Definition 3.3.6 Define $\mathcal{F}_{\infty}^{i}$ to be the full subcomplex of $\mathcal{B}^{i}(g)$ with vertices in $\left\{A_{1}, A_{2}, A_{3}, A_{4}\right\}$. Define $\mathcal{F}_{k}^{1}$ to be the full subcomplex of $\mathcal{B}^{1}(g)$ with vertices in $H_{k} \cup\left\{A_{1}, A_{2}, A_{3}, A_{4}\right\}$, where $H_{k}=\left\langle a_{k}, b_{k}, \ldots, a_{g}, b_{g}\right\rangle \subseteq H$.

To define $\mathcal{F}_{k}^{2}$, let $\tilde{\mathcal{F}}_{k}^{2}$ be the full subcomplex of $\mathcal{B}^{2}(g)$ with vertices in $\left(a_{1}+H_{k}\right) \cup$ $\left\{A_{1}, A_{2}, A_{3}, A_{4}\right\}$, and set $\mathcal{F}_{k}^{2}=\operatorname{star}_{\mathcal{B}^{2}(g)}\left(a_{1}\right) \cap \tilde{\mathcal{F}}_{k}^{2}$ for $k \geq 2$. For $k=1$ we set $\mathcal{F}_{1}^{2}=\mathcal{O B}^{a_{1}}{ }_{\mathrm{gcd}=1}(g) \cup \mathcal{F}_{2}^{2}$; see Definition 2.4.4.

As a general convention $\mathcal{F}_{5+1}^{i}=\mathcal{F}_{\infty}^{i}$. (We do not call it $\mathcal{F}_{6}^{i}$ since it does not agree with the definition of $\mathcal{F}_{k}^{i}$ for $k=6$.)

Lemma 3.3.7 Let $i \in\{1,2\}$ and $k \leq 5$. To each $s$-simplex $\mathfrak{w} \in \mathcal{F}_{k}^{i} \backslash \mathcal{F}_{k+1}^{i}$, we can assign a simplex $\mathfrak{w}^{*}=\left\{w_{1}, \ldots, w_{g-3+i-s}\right\} \in \mathcal{F}_{k+1}^{i}$ such that:

(i) $\mathfrak{w} * \mathfrak{w}^{*}$ is a simplex in $\mathcal{F}_{k}^{i}(g)$.

(ii) $w_{j}=A_{j}$ for $1 \leq j \leq k-1$.

(iii) If $\mathfrak{v} \subseteq \mathfrak{w}$ with $\mathfrak{v} \in \mathcal{F}_{k+1}^{i}(g)$, then $\mathfrak{v} * \mathfrak{w}^{*} \in \mathcal{F}_{k+1}^{i}(g)$.

(iv) For $i=2$ and $k=0$, write $\mathfrak{w}=\left\{\mathfrak{w}_{0}, \ldots, \mathfrak{w}_{s}\right\} \in \mathcal{B}^{2}(g)$, then $\operatorname{rk}^{b_{1}}\left(\mathfrak{w}_{0}\right)=$ $\mathrm{rk}^{b_{1}}\left(w_{j}\right)$ for all $j$.

Proof We set $w_{j}=A_{j}$ for $1 \leq j \leq k-1$, and now define the remaining $w_{j}$ for $k \leq j \leq g-3+i-s$. Set $m=\max (k+1,2)$, and let $S=S\left(\operatorname{pr}_{m}(\mathfrak{w})\right)$. Then use Proposition 2.2.9 to obtain a dual summand $D$ in $H_{m}$, and a symplectic subspace $T$ such that $(S \oplus D) \oplus T=H_{m}$. We want to choose $w_{j}$ using the basis vectors $\left\{t_{1}, \ldots, t_{n}\right\}$ for an isotropic summand of $T$. A dimension count shows $\operatorname{dim} T \geq 2(g-1+i-s-m)$. For $i=1$, we put $w_{j}=t_{j}$, and are done.

Now let $i=2$. We put $w_{j}=a_{1}+t_{j}$ when $k \geq 2$. When $k \leq 1$ this may not work since $a_{1}+t_{j}$ may not be orthogonal to $\mathfrak{w}$. But by Lemma 2.5.6 we can choose $u \in D$ such that $a_{1}+t b_{1}+u \perp \mathfrak{w}$ for $t \in \mathbb{Z}$ as in (19). Then set $w_{j}=a_{1}+t b_{1}+u+t_{j}$. (iii) is automatic except for $k=0$, where it follows from Remark 2.2.7(ii), using $\operatorname{gcd}_{2}(\mathfrak{v})=1$ and the vectors of $\operatorname{pr}_{2}(\mathfrak{v})$ are in $S$, while $w_{j} \in D \oplus T$. (iv) follows from the choice of $t$. 
Assuming $g \geq 8-i$, we always get (at least) a 4 -simplex $x^{*}=\left\{x_{1}, x_{2}, x_{3}, x_{4}, x_{5}\right\} \in$ $\mathcal{F}_{k+1}^{i}$ assigned to each vertex $x \in \mathcal{F}_{k}^{i}$. We emphasize that for each vertex $x$ we choose $x^{*}$ once and for all.

\subsection{Constructing the Morse vector field}

So we must prove Theorem 3.2.3. The easy part is degree 0 and 1 :

Proposition 3.4.1 Let $i \in\{1,2\}$. There is a Morse vector field on $E_{*}^{i}$ that spans $E_{0}^{i}, E_{1}^{i}$, and where $\mathcal{C}_{2}$ is given by $\mathcal{C}_{2}=\mathcal{C}_{2}(\infty) \oplus \mathcal{C}_{2}^{\prime}$.

Here, $\mathcal{C}_{2}(\infty)=\mathcal{R}\left(A_{2}\right) \oplus \mathcal{R}\left(A_{3}\right) \oplus \mathcal{R}\left(A_{4}\right)$ in the notation of Lemma 3.3.4 with $\mathfrak{w}=b^{i}$. And letting $x^{*}=\left\{x_{1}, x_{2}, x_{3}, x_{4}\right\}$ as in Lemma 3.3.7,

$$
\begin{aligned}
\mathcal{C}_{2}^{\prime}= & \bigoplus_{\substack{x \in \mathcal{B}^{i}(g)^{(0)} \\
x \notin\left\{A_{1}, \ldots, A_{4}\right\}}}\left(\wedge^{3}\left\langle x, x_{1}, b^{i}\right\rangle_{\mathbb{Q}}^{\perp} \oplus\left\langle y_{1}\right\rangle \wedge \wedge^{2}\left\langle x, x_{2}, b^{i}\right\rangle_{\mathbb{Q}}^{\perp}\right. \\
& \left.\oplus\left\langle y_{1} \wedge y_{2}\right\rangle \wedge\left\langle x, x_{3}, b^{i}\right\rangle_{\mathbb{Q}}^{\perp} \oplus\left\langle y_{1} \wedge y_{2} \wedge y_{3}\right\rangle\right),
\end{aligned}
$$

where $y_{j}$ denotes a choice of dual vector to $x_{j}$.

Proof In degree 0 , we have $E_{0}^{i}=\bigwedge^{3}\left\langle b^{i}\right\rangle_{\mathbb{Q}}^{\perp}$. We use Corollary 3.3.3 on $\mathfrak{w}=b^{i}$ with $w_{j}=A_{j}$ and $u_{j}=B_{j}$ for $j=1,2,3,4$; see (35) and (36). This yields a vector field that spans $E_{0}^{i}$, and no gradient paths at all, since $d=\partial_{0}$.

Now consider degree 1. $\mathcal{C}_{1}$ can be read off from Lemma 3.3.2 (here, $i=1$ ):

$$
\begin{aligned}
\mathcal{C}_{1} & =\bigwedge^{3}\left\langle A_{1}\right\rangle_{\mathbb{Q}}^{\perp} \oplus\left\langle B_{1}\right\rangle \wedge \bigwedge^{2}\left\langle A_{2}\right\rangle_{\mathbb{Q}}^{\perp} \oplus\left\langle B_{1} \wedge B_{2}\right\rangle \wedge\left\langle A_{3}\right\rangle_{\mathbb{Q}}^{\perp} \oplus\left\langle B_{1} \wedge B_{2} \wedge B_{3}\right\rangle \\
& \subseteq \bigwedge^{3}\left\langle A_{1}\right\rangle_{\mathbb{Q}}^{\perp} \oplus \wedge^{3}\left\langle A_{2}\right\rangle_{\mathbb{Q}}^{\perp} \quad \oplus \bigwedge^{3}\left\langle A_{3}\right\rangle_{\mathbb{Q}}^{\perp} \quad \oplus \wedge^{3}\left\langle A_{4}\right\rangle_{\mathbb{Q}}^{\perp}
\end{aligned}
$$

Consequently, $\mathcal{R}_{1}$ has to be the rest of $E_{1}^{i}=\bigoplus_{x \in \mathcal{B}^{i}(g)^{(0)}} \bigwedge^{3}\left\langle x, b^{i}\right\rangle_{\mathbb{Q}}^{\perp}$.

For $A_{1}, A_{2}, A_{3}, A_{4}$, we use Corollary 3.3.5. In general, for each $x \in \mathcal{B}^{i}(g)^{(0)}, x \neq$ $A_{1}, A_{2}, A_{3}, A_{4}$, we choose the vector field on $\bigwedge^{3}\left\langle x, b^{i}\right\rangle_{\mathbb{Q}}^{\perp}$ as in Corollary 3.3.3, using the four vectors of the simplex $x^{*}=\left\{x_{1}, x_{2}, x_{3}, x_{4}\right\}$ provided by Lemma 3.3.7. Then we get a vector field spanning $E_{1}^{i}$ with $\mathcal{C}_{2}$ as stated.

We now argue why there are no infinite gradient paths starting in $\mathcal{R}_{1}$. By construction, if there is $\left(b, b^{\prime}\right) \rightarrow\left(d, d^{\prime}\right)$ where $\operatorname{simp}(b)=x$, then $\operatorname{simp}(d)=x_{j}$ for some $j=$ $1,2,3,4$. We have ensured that if $x \in \mathcal{F}_{k}^{i}$ then $x_{j} \in \mathcal{F}_{k+1}^{i}$, and if $k=5$, then $x_{j}=A_{j}$. Thus any gradient path reaches some $A_{j}(j=1,2,3,4)$ in at most five steps. And if $\operatorname{simp}(b)=A_{j}$, by construction either $d \in \mathcal{C}_{1}$, or $\operatorname{simp}(d)=A_{k}$ with $k<j$. We see any gradient path has length $\leq 9$. 
Now we will extend the vector field defined in Proposition 3.4.1 to degree 2 by induction in the filtration $\mathcal{F}_{k}^{i}$. The induction hypothesis is $A(k)$ :

$A(k)$ : There is a Morse vector field $V_{*}^{i}$ on $E_{*}^{i}$ that spans $E_{0}^{i}, E_{1}^{i}$, and $E_{2}^{i}(k):=$ $\bigoplus_{\mathfrak{w} \in \mathcal{F}_{k}^{i}(g)^{(1)}} \bigwedge^{3}\left\langle\mathfrak{w}, b^{i}\right\rangle_{\mathbb{Q}}^{\perp}$, for $i=1,2$.

Using Corollary 3.3.5, it is easy to prove the base case of the induction, $A(\infty)$. We now inductively assume $A(k+1), k \in\{1,2,3,4,5\}$ (interpreting $5+1$ as $\infty$ ), and we wish to prove $A(k)$. This will be done in the next lemmas.

Lemma 3.4.2 We can extend the Morse vector field $V_{2}^{i}$ so it spans

$$
\bigoplus_{x \in \mathcal{F}_{k}^{i}(g)^{(0)}} \bigoplus_{j=1}^{4} \bigwedge^{3}\left\langle x, x_{j}, b^{i}\right\rangle_{\mathbb{Q}}^{\perp} \subseteq E_{2}^{i}(k) .
$$

Proof Note that (37) is precisely the part of $E_{2}^{i}(k)$ which intersects $\mathcal{C}_{2}$ nontrivially. We know $\mathcal{C}_{2}$ from Proposition 3.4.1, $\mathcal{C}_{2}=\mathcal{C}_{2}^{\prime} \oplus \mathcal{C}_{2}(\infty)$. The vector field on the summands of $E_{2}^{i}(k)$ intersecting $\mathcal{C}_{2}(\infty)$ is the induction start, so we must extend it to the summands intersecting $\mathcal{C}_{2}^{\prime}$. Apply Corollary 3.3.5 for each $x \in \mathcal{F}_{k}^{i}(g)^{(0)} \backslash$ $\left\{A_{1}, \ldots, A_{4}\right\}$ to construct the vector field. There are no infinite gradient paths by induction in $k$, precisely as in the proof of Proposition 3.4.1.

For now, take a fixed vertex $x \in \mathcal{F}_{k}^{i} \backslash \mathcal{F}_{k+1}^{i}$. Define $E^{i}(x) \subseteq E_{2}^{i}(k)$ by

$$
E^{i}(x)=\bigoplus_{\substack{x^{\prime} \in \mathcal{F}_{k+1}^{i}(g)^{(0)}: \\\left\{x, x^{\prime}\right\} \in \mathcal{F}_{k}^{i}(g)}} \bigwedge^{3}\left\langle x, x^{\prime}, b^{i}\right\rangle_{\mathbb{Q}}^{\perp}
$$

We will choose a basis of $E^{i}(x)$ in the next lemmas. Our method does not choose a basis of each summand $\bigwedge^{3}\left\langle x, x^{\prime}, b^{i}\right\rangle_{\mathbb{Q}}^{\perp}$ at a time, instead mixing them up; this is so that we can argue there are no infinite gradient paths.

Lemma 3.4.3 For a fixed vertex $x \in \mathcal{F}_{k}^{i} \backslash \mathcal{F}_{k+1}^{i}$, there is a Morse vector field extending $V_{*}^{i}$ that spans a subset $A_{x}^{i} \subseteq E^{i}(x)$ ( $A_{x}^{i}$ is defined in the proof).

Proof We can write $E^{i}(x)=\bigoplus_{x^{\prime} \in \mathcal{F}_{k+1}^{i}(g)^{(0)}}\left\langle z \mid\left(z, x^{\prime}\right) \in S\right\rangle$, where $S$ is the following index set:

$$
S=\left\{\left(z, x^{\prime}\right) \in \bigwedge^{3} H_{\mathbb{Q}} \times \mathcal{F}_{k+1}^{i}(g)^{(0)} \mid\left\{x, x^{\prime}\right\} \in \mathcal{F}_{k+1}^{i}(g)^{(1)}, z \in \bigwedge^{3}\left\langle x, x^{\prime}, b^{i}\right\rangle_{\mathbb{Q}}^{\perp}\right\}
$$


We filter $E^{i}(x)$ by subsets $F_{0} \subseteq F_{1} \subseteq F_{2} \subseteq \cdots \subseteq E^{1}(x)$, by inductively defining $S_{0} \subseteq S_{1} \subseteq \cdots \subseteq S$, and setting $F_{j}=\bigoplus_{x^{\prime} \in \mathcal{F}_{k+1}^{i}}\left\langle z \mid\left(z, x^{\prime}\right) \in S_{j}\right\rangle$. For the induction start, define $S_{0}=\left\{\left(z, x^{\prime}\right) \in S \mid x^{\prime} \in\left\{x_{1}, x_{2}, x_{3}, x_{4}\right\}\right\}$, and note $F_{0}$ is already handled in the proof of Lemma 3.4.2. For the induction step, assume we have defined $F_{j-1}$ and its basis $B_{j-1}$. Then set

$$
\begin{aligned}
S_{j}=\left\{\left(z, x^{\prime}\right) \in S \mid \exists x^{\prime \prime}:\left(z, x^{\prime \prime}\right) \in F_{j-1},\left\{x, x^{\prime}, x^{\prime \prime}\right\}\right. & \in \mathcal{F}_{k+1}^{i}(g)^{(2)}, \\
& \left.z \in \bigwedge^{3}\left\langle x, x^{\prime}, x^{\prime \prime}, b^{i}\right\rangle_{\mathbb{Q}}^{\perp}\right\} .
\end{aligned}
$$

Choose a basis $B_{j}$ for $F_{j}$ by extending $B_{j-1}$ by suitable vectors $z$ with $\left(z, x^{\prime}\right) \in S_{j}$. Set $A_{x}^{i}=\bigcup_{i=1}^{\infty} F_{j} \subseteq E^{i}(x)$. We get the basis $B_{x}=\bigcup_{j=1}^{\infty} B_{j}$ for $A_{x}^{i}$. To define the vector field, for $z \in B_{j}$, set $\left.\operatorname{simp}(c(z))\right)=\left\{x, x^{\prime}, x^{\prime \prime}\right\}$ with $x^{\prime \prime}$ as in (39). The vector field spans $A_{x}^{i}$ by construction.

We prove that there are no infinite gradient paths by induction in $j$. The induction start is Lemma 3.4.2. A gradient path from $z \in B_{j}$ leads to either a vector with simplex $\left\{x^{\prime}, x^{\prime \prime}\right\} \in \mathcal{F}_{k+1}^{i}$ or to $F_{j-1}$; and there are no infinite gradient paths starting there by induction in $k$ or $j$, respectively.

Proposition 3.4.4 If $g \geq 8-i, A_{x}^{i}=E^{i}(x)$.

This is a major step, and the differences between $i=1,2$ become so pronounced that we handle them separately, in the next two subsections. First, though, we observe it suffices to prove the following:

Lemma 3.4.5 Given $x^{\prime} \in \mathcal{F}_{k+1}^{1}$ such that $\left\{x, x^{\prime}\right\}$ is a simplex, there exist a basis $\bar{B}\left(x^{\prime}\right)$ for $\bigwedge^{3}\left\langle x, x^{\prime}\right\rangle_{\mathbb{Q}}^{\perp}$ such that for all $z \in \bar{B}\left(x^{\prime}\right)$ there is $s \in \mathbb{N}$ with $\left(z, x^{\prime}\right) \in F_{s}$.

Remark 3.4.6 For $x_{1}, \ldots, x_{5}$ associated to $x$ from Lemma 3.3.7, there are bases $\bar{B}\left(x_{m}\right)$ satisfying Lemma 3.4.5: Given any $z \in \bigwedge^{3}\left\langle x, x_{m}, b_{1}\right\rangle_{\mathbb{Q}}^{\perp}$, from the definition it is easy to see that $\left(x_{m}, z\right) \in F_{1}$, where $m=1,2,3,4,5$.

Lemma 3.4.7 Assuming Lemma 3.4.5, $A(k)$ holds.

Proof We have then by Lemma 3.4.3 a Morse vector field spanning

$$
E^{i}(k, k+1):=\bigoplus_{x \in \mathcal{F}_{k}^{i} \backslash \mathcal{F}_{k+1}^{i}} E^{i}(x)=\bigoplus_{\substack{\left\{x, x^{\prime}\right\} \in \mathcal{F}_{k}^{i}(g)^{(1)}: \\ x \in \mathcal{F}_{k}^{i} \backslash \mathcal{F}_{k+1}^{i}, x^{\prime} \in \mathcal{F}_{k+1}^{i}}} \bigwedge^{3}\left\langle x, x^{\prime}, b^{i}\right\rangle_{\mathbb{Q}}^{\perp},
$$

All we are missing are the cases $x, x^{\prime} \in \mathcal{F}_{k}^{i} \backslash \mathcal{F}_{k+1}^{i}$ and $x, x^{\prime} \in \mathcal{F}_{k+1}^{i}$ but $\left\{x, x^{\prime}\right\} \in$ $\mathcal{F}_{k}^{i} \backslash \mathcal{F}_{k+1}^{i}$, which we handle in the same way: We take $\left\{x, x^{\prime}\right\}^{*}=\left\{v_{1}, v_{2}, v_{3}, v_{4}\right\}$ 
from Lemma 3.3.7. Construct the vector field as in Corollary 3.3.5. Given a gradient path starting in some vector with simplex $\left\{x, x^{\prime}\right\}$, it can either lead to $\left\{x, v_{i}\right\}$ or $\left\{x^{\prime}, v_{i}\right\}$, both of which are simplices for vectors in $E^{i}(k, k+1)$ in the first case, or in $E_{2}^{i}(k+1)$ in the second case by Lemma 3.3.7(iii), and no infinite gradient paths start there, in the latter case by $A(k+1)$.

Pending the proof of Lemma 3.4.5, we have now proved Theorem 3.2.3.

Now we prove Proposition 3.2.4, that this vector field extends to a Morse vector field on $E_{*, 1}^{1}$ :

Proof of Proposition 3.2.4 For a simplex $\mathfrak{w} \in \mathcal{B}(g)$, we write $\mathcal{O}(\mathfrak{w}) \in \mathcal{B}^{i}(g)$ for the simplex with the same vertices placed in ascending order and $\overline{\mathcal{O}}(\mathfrak{w})$ for the descending order. We proceed by induction in the filtration degree $k$ to show that given a simplex $(x, y)=\overline{\mathcal{O}}(x, y)$ with $(y, x) \in \mathcal{F}_{k}^{i}$ we can extend the vector field by a basis for the summand $\bigwedge^{3}\left\langle x, y, b^{i}\right\rangle \stackrel{\perp}{\mathbb{Q}}$ indexed by $(x, y)$.

Let $(y, x) \in \mathcal{F}_{k}^{i}(g)$, and assume one of $x$ and $y$, say $y$, is in $\mathcal{F}_{k+1}^{i}(g)$. By Lemma 3.3.7, we get a 4 -simplex $(y, x)^{*}=\mathcal{O}\left(w_{1}, w_{2}, w_{3}, w_{4}\right) \in \mathcal{F}_{k+1}^{i}$ and by Lemma 3.3 .2 we get the basis $B(x, y)$ for $\bigwedge^{3}\left\langle x, y, b^{i}\right\rangle_{\mathbb{Q}}^{\perp}$ such that for each $z \in B(x, y)$ there is $w_{z}=w_{j}$ for a $j \in\{1,2,3,4\}$ with $z \in \bigwedge^{3}\left\langle x, y, w_{z}, b^{i}\right\rangle_{\mathbb{Q}}^{\perp}$. Then let $\operatorname{simp}(c(z))=$ $\left(\mathcal{O}\left(x, w_{z}\right), y\right)$. The other two differentials from $\left(\mathcal{O}\left(x, w_{z}\right), y\right)$ land in summands indexed by, respectively, $\mathcal{O}\left(x, w_{z}\right) \in E_{2}^{i}$, and $\left(w_{z}, y\right)$ with $\mathcal{O}\left(w_{z}, y\right) \in \mathcal{F}_{k+1}^{i}$ by Lemma 3.3.7(iii). So we create no infinite gradient paths, in the first case by the assumption of the lemma, and in the second case by induction in $k$.

We must show that the vectors $c(z)$ thus defined are linearly independent. The only 1 -simplices besides $(x, y)$ which could get paired with $\left(\mathcal{O}\left(x, w_{z}\right), y\right)$ in the vector field are $\mathcal{O}\left(x, w_{z}\right)$ and $\left(w_{z}, y\right)$. The former is in $\mathcal{B}^{i}(g)$ so its partner is likewise in $\mathcal{B}^{i}(g)$ by assumption, and by inspection, it is easy to see that the latter cannot get paired with $\left(\mathcal{O}\left(x, w_{z}\right), y\right)$ by the method above.

To finish the induction step, assume $x, y \in \mathcal{F}_{k}^{i} \backslash \mathcal{F}_{k+1}^{i}$, and proceed exactly as above. The simplices $\mathcal{O}\left(x, w_{z}\right)$ and $\left(w_{z}, y\right)$ now have one vertex in $\mathcal{F}_{k+1}^{i}$, and so are dealt with above. The induction start deals with $x, y$ among $A_{1}, \ldots, A_{4}$ by a similar method, using as $\left\{w_{1}, \ldots, w_{4}\right\}$ the four remaining among $A_{1}, \ldots, A_{6}$.

\subsection{Finishing the proof of Theorem 3.2.3 for $i=1$}

In this section, $i=1$. We must prove Lemma 3.4.5. In light of Remark 3.4.6, we will deduce the existence of $\bar{B}\left(x^{\prime}\right)$ from that of $\bar{B}\left(x_{m}\right)$, where $m=1,2,3,4,5$. To do this, we need some preliminary Lemmas. 
Lemma 3.5.1 Suppose $\left\{x, x^{\prime}\right\} \in \mathcal{F}_{k}^{1}(g)$ satisfies $x, x^{\prime} \notin\left\{a_{1}, \ldots, a_{k-1}\right\}$. Assume we are given $v_{1}, v_{2}, v_{3}, v_{4} \in \mathcal{F}_{k+1}^{1}(g)^{(0)}$ such that $\left\{x, v_{1}, v_{2}, v_{3}, v_{4}\right\} \in \mathcal{F}_{k}^{1}(g)$, and $\operatorname{gcd}\left(x, x^{\prime}, v_{1}, v_{2}, v_{3}, v_{4}\right)>0$, and $v_{j}=a_{j}$ for $1 \leq j \leq k-1$. Then:

(i) There is a $\mathbb{Q}$-basis $B=B\left(x^{\prime}\right)$ for $\left\langle x, x^{\prime}\right\rangle^{\perp}$ that satisfies:

(a) $\left\{a_{1}, b_{1}, \ldots, a_{k-1}, b_{k-1}\right\} \subseteq B \subseteq \mathcal{F}_{k}^{1}(g)^{(0)} \cup\left\{b_{1}, \ldots, b_{k-1}\right\}$.

(b) $\forall z \in B:\left|\left\{1 \leq j \leq 4 \mid z \perp v_{j}\right\}\right| \geq 3$.

(ii) There is a basis $\bar{B}=\bar{B}\left(x^{\prime}\right)$ for $\bigwedge^{3}\left\langle x, x^{\prime}\right\rangle_{\mathbb{Q}}^{\perp}$ such that for each $\bar{z} \in \bar{B}$ there is $1 \leq j \leq 4$ with $\bar{z} \in \bigwedge^{3}\left\langle x, v_{j}\right\rangle_{\mathbb{Q}}^{\perp}$.

Proof Write $H_{<k}=\left\langle a_{1}, b_{1}, \ldots, a_{k-1}, b_{k-1}\right\rangle$. By assumption, $x, x^{\prime} \in H_{k}$ therefore $\left\langle x, x^{\prime}\right\rangle^{\perp}=H_{<k} \oplus V_{k}$, where $V_{k} \subseteq H_{k}$. We choose $B=\left\{a_{1}, b_{1}, \ldots, a_{k-1}, b_{k-1}\right\} \cup B_{k}$, where $B_{k} \subseteq \mathcal{F}_{k}^{1}(g)^{(0)}$ is a basis of $V_{k}$ that satisfies (b).

To construct $B_{k}$, consider $\left\langle x, x^{\prime}, v_{k}, \ldots, v_{4}\right\rangle^{\perp}$. Since $v_{i} \in H_{k}$ for $i \geq k$, we have again $\left\langle x, x^{\prime}, v_{k}, \ldots, v_{4}\right\rangle^{\perp}=H_{<k} \oplus V_{k}^{\prime}$, where $V_{k}^{\prime} \subseteq V_{k}$. Take a basis $B_{k}^{\prime}$ of $V_{k}^{\prime}$. Now we choose a type of "dual vectors" to $v_{k}, \ldots, v_{4}$, as follows: Consider, for $k \leq j \leq 4$, the space $\left\langle x, x^{\prime}, v_{k}, \ldots, \widehat{v}_{j}, \ldots, v_{4}\right\rangle^{\perp}=H_{<k} \oplus V_{k}^{\prime}(j)$, where $V_{k}^{\prime} \subseteq V_{k}^{\prime}(j)$. Choose $u_{j} \in V_{k}^{\prime}(j)$ such that it maps to a simple vector under the quotient map $V_{k}^{\prime}(j) \longrightarrow$ $V_{k}^{\prime}(j) / V_{k}^{\prime}$. Then $u_{j} \in \mathcal{F}_{k}^{1}(g)$, and by construction, $B_{k}:=B_{k}^{\prime} \cup\left\{u_{k}, \ldots, u_{4}\right\}$ is a $\mathbb{Q}$-basis of $V_{k}$ that satisfies (b). This shows (i), and (ii) follows immediately.

The following gives us a way to ensure that a basis element $z_{1} \wedge z_{2} \wedge z_{3} \in \wedge^{3}\left\langle x, x_{j}\right\rangle_{\mathbb{Q}}^{\perp}$ can be "carried along" to $\bigwedge^{3}\left\langle x, x^{\prime}\right\rangle \frac{\perp}{\mathbb{Q}}$. We owe the idea to A Putman, from his master class The Torelli group at Aarhus University, 2008.

Definition 3.5.2 Let $i \in\{1,2\}$, and let $0 \leq k \leq 5$. Let $x \in \mathcal{F}_{k}^{i} \backslash \mathcal{F}_{k+1}^{i}$. For $z_{1}, \ldots, z_{n} \in$ $\langle x\rangle_{\mathbb{Q}}^{\perp}$, set $Z=\left\langle z_{1}, \ldots, z_{n}\right\rangle_{\mathbb{Q}}^{\perp}$, and let $W^{i}$ denote the full subcomplex of $\mathcal{B}^{i}(g)$ with vertices in $Z$. Define the complex $\mathcal{B}_{Z, x, k}^{i}(g)$ to be

$$
\mathcal{B}_{Z, x, k}^{i}(g)=\left\{\mathfrak{v} \in \mathcal{F}_{k+1}^{i} \cap W^{i} \mid\{x, \mathfrak{v}\} \in \mathcal{F}_{k}^{i}\right\} .
$$

Note, for a simplex $\mathfrak{v} \in \mathcal{B}_{Z, x, k}^{1}(g)$, if $n=3$, we have $z_{1} \wedge z_{2} \wedge z_{3} \in \wedge^{3}\langle x, \mathfrak{v}\rangle \frac{\perp}{\mathbb{Q}}$.

Lemma 3.5.3 Let $n+4 \leq g$. Assume we are given a basis $B$ of $\left\langle x, x^{\prime}\right\rangle_{\mathbb{Q}}^{\perp}$ that satisfies Lemma 3.5.1(i)(a), and assume $z_{1}, \ldots, z_{n} \in B$. Then $\mathcal{B}_{Z, x, k}^{1}(g)$ is connected.

Proof First, assume $a_{j} \in Z$ for some $1 \leq j<k \leq 5$. Then $a_{j} \in \mathcal{B}_{Z, x, k}^{1}(g)$; moreover, for any $v \in \mathcal{B}_{Z, x, k}^{1}(g)^{(0)} \backslash\left\{a_{j}\right\}$ we have $\left\{a_{j}, v\right\} \in \mathcal{B}_{Z, x, k}^{1}(g)^{(1)}$. Thus $\mathcal{B}_{Z, x, k}^{1}(g)$ is connected in this case. Note this takes care of $n<k-1$. 
Now let $n \geq k-1$. We can assume $a_{j} \notin Z$ for all $j<k$. Since $z_{m} \in B$, we must have $z_{j}=b_{j}$ for $1 \leq j \leq k-1$. Then $\mathcal{F}_{k+1}^{1}(g) \cap Z=\mathcal{F}_{k+1}^{1}(g) \cap\left\langle z_{k}, \ldots, z_{n}\right\rangle_{\mathbb{Q}}^{\perp} \backslash$ $\left\{a_{1}, \ldots, a_{k-1}\right\}$. So if we set $Z^{\prime}=\left\langle z_{k}, \ldots, z_{n}\right\rangle_{\mathbb{Q}}^{\perp} \backslash\left\{a_{1}, \ldots, a_{k-1}\right\}$, then $\mathcal{B}_{Z, x, k}^{1}(g)=$ $\mathcal{B}_{Z^{\prime}, x, k}^{1}(g)$. Thus it suffices to show $\mathcal{B}_{Z^{\prime}, x, k}^{1}(g)$ is connected.

For now, assume $n \geq k$. Given $v_{1}, v_{2} \in \mathcal{B}_{Z^{\prime}, x, k}^{1}(g)^{(0)}$, we will show there is a path $v_{1} \rightarrow w \rightarrow v_{2}$ in $\mathcal{B}_{Z^{\prime}, x, k}^{1}(g)$. Let $V^{1}=\left\langle v_{1}, v_{2}, x\right\rangle$ and $V^{2}=\left\langle z_{k}, \ldots, z_{n}\right\rangle$. The idea is that $w$ need not be independent of $z_{k}, \ldots, z_{n}$, only orthogonal to them. Let $S V_{k+1}^{j}$ be the smallest summand in $H_{k+1}$ containing $V_{k+1}^{j}=\operatorname{pr}_{k+1}\left(V^{j}\right)$. Take a dual summand $D^{1}$ (cf Proposition 2.2.9) to $S V_{k+1}^{1}$ in $H_{k+1}$, ie there is $T^{1}$ so that $\left(S V_{k+1}^{1} \oplus D^{1}\right) \oplus T^{1}=H_{k+1}$. Now take a dual $D^{2}$ to $S V_{k+1}^{2,1}=S\left(\operatorname{pr}_{T_{1}}\left(V_{k+1}^{2}\right)\right)$ in $T^{1}$, so obtaining $T^{2} \perp V^{1}+V^{2}$ with

$$
\left(S V_{k+1}^{1} \oplus D^{1}\right) \oplus\left(S V_{k+1}^{2,1} \oplus D^{2}\right) \oplus T^{2}=H_{k+1} .
$$

Since $n \geq k$, if $S V_{k+1}^{2,1}=0$, then $\operatorname{dim} T^{2} \geq 2(g-k-3) \geq 2$. Thus, any simple vector $w \in T^{2}$ gives a path $v_{1} \rightarrow w \rightarrow v_{2}$ in $\mathcal{B}_{Z^{\prime}, x, k}^{1}(g)$. If $S V_{k+1}^{2,1} \neq 0$, then choose $w^{\prime} \in S V_{k+1}^{2,1}$, and use the dual basis for $D^{2}$ to modify $w^{\prime} \rightsquigarrow w$ such that $w \perp z_{i}$, $i=k, \ldots, 3$. Then $w \in\left(S V_{k+1}^{2,1} \oplus D^{2}\right)$, and so forms a simplex with $x, v_{1}, v_{2}$, since they are in $S V_{k+1}^{1}$. This proves the Lemma for $n \geq k$.

Now let $n=k-1 \leq 4$, ie $Z^{\prime}=H \backslash\left\{a_{1}, \ldots, a_{k-1}\right\}$. Again given $v_{1}, v_{2} \in \mathcal{B}_{Z^{\prime}, x, k}^{1}(g)^{(0)}$, set $\bar{x}=\operatorname{pr}_{H_{k+1}}(x) / \operatorname{gcd}\left(\operatorname{pr}_{H_{k+1}}(x)\right)$. Consider $\bar{V}^{j}=\left\langle v_{j}, \bar{x}\right\rangle$ for $j=1$, 2, project to $H_{k+1}$ and take the dual as above, $\left(S \bar{V}_{k+1}^{j} \oplus D^{j}\right) \oplus \bar{T}_{1}^{j}=H_{k+1}$, and the dimension argument above gives $\bar{T}_{1}^{j} \neq 0$. We obtain a simple vector $w_{j} \in \bar{T}_{1}^{j}$. Define the subcomplex $\overline{\mathcal{F}}_{k+1}(g) \subseteq \mathcal{F}_{k+1}^{1}(g)$ of simplices with vertices in $H_{k+1}$. Thus, $w_{j} \in \operatorname{link}_{\overline{\mathcal{F}}_{k+1}(g)}(\bar{x})$. Now, $\overline{\mathcal{F}}_{k+1}(g) \cong \mathcal{B}(g-k)$ via $H_{k+1} \cong H(g-k)$. Then by Proposition 2.3.3, $\operatorname{link}_{\overline{\mathcal{F}}_{k+1}(g)}(\bar{x})$ is connected for $g \geq k+3=n+4$, so there is a path from $w_{1}$ to $w_{2}$. This yields the desired path in $\mathcal{B}_{Z^{\prime}, x, k}^{1}(g)$, namely $v_{1} \rightarrow w_{1} \rightarrow \cdots \rightarrow w_{2} \rightarrow v_{2}$.

Proof of Lemma 3.4.5 for $i=1$ As usual $x_{1}, \ldots, x_{5}$ is the vectors associated to $x$ from Lemma 3.3.7. Let $\bar{B}\left(x^{\prime}\right)$ be the basis for $\bigwedge^{3}\left\langle x, x^{\prime}\right\rangle_{\mathbb{Q}}^{\perp}$ from Lemma 3.5.1, where $v_{1}, \ldots, v_{4}$ are four among $x_{1}, \ldots, x_{5}$. So for each $z=z_{1} \wedge z_{2} \wedge z_{3} \in \bar{B}$ there is $1 \leq j \leq 5$ with $z \in \bigwedge^{3}\left\langle x, x_{j}\right\rangle_{\mathbb{Q}}^{\perp}$. For each $z \in \bar{B}$, set $Z=\left\langle z_{1}, z_{2}, z_{3}\right\rangle^{\perp}$, and consider the complex $\mathcal{B}_{Z, x, k}^{1}(g)$ from Lemma 3.5.3, which is connected. We see $x^{\prime}, x_{j} \in \mathcal{B}_{Z, x, k}^{1}(g)$, so there is a path in $\mathcal{B}_{Z, x, k}^{1}(g)$ connecting them, $x_{j}=v_{0} \rightarrow v_{1} \rightarrow \cdots \rightarrow v_{\ell}=x^{\prime}$. Then $\left(z, x^{\prime}\right) \in F_{\ell+1}$ by the lemma below, since $\left(z, x_{m}\right) \in F_{1}$ by Remark 3.4.6.

Lemma 3.5.4 Let $z=z_{1} \wedge z_{2} \wedge z_{3}$ for $z_{j} \in \bigwedge^{3}\langle x, v\rangle_{\mathbb{Q}}^{\perp}$. If there is a path $v=v_{0} \rightarrow$ $v_{1} \rightarrow \cdots \rightarrow v_{\ell}=x^{\prime}$ in $\mathcal{B}_{Z, x, k}^{i}(g)$, and $(z, v) \in F_{m}$, then $\left(z, x^{\prime}\right) \in F_{m+\ell}$.

Proof A straightforward induction shows $\left(z, v_{j}\right) \in F_{j+m}$ for $j=1, \ldots, \ell$. 


\subsection{Finishing the proof of Theorem 3.2.3 for $i=2$}

In this section, $i=2$. Recall we write $S(V)=S\left(\operatorname{pr}_{2}(V)\right)$, where $V \subseteq H$. To prove Lemma 3.4.5, we use the same strategy as for $i=1$, but the details are more complicated. First, we establish versions of Lemma 3.5.1 and Lemma 3.5.3:

Remark 3.6.1 For $i=2$, we apply Lemma 3.5.1 to $H_{2}=\operatorname{pr}_{2} H$ to get a basis $B_{2}\left(x^{\prime}\right)$ of $\operatorname{pr}_{2}\left(\left\langle\operatorname{pr}_{2}(x), \operatorname{pr}_{2}\left(x^{\prime}\right)\right\rangle_{\mathbb{Q}}^{\perp}\right)$ with (a) replaced by

$$
\left\{a_{2}, b_{2}, \ldots, a_{k-1}, b_{k-1}\right\} \subseteq B \subseteq \mathcal{F}_{\max (k, 2)}^{1}(g)^{(0)} \cup\left\{b_{2}, \ldots, b_{k-1}\right\} .
$$

A modification for $k=1$, needed to apply Corollary 3.6.2 below: If we assume $\operatorname{gcd}_{2}\left(x, x^{\prime}, v_{1}, v_{2}, v_{3}, v_{4}\right)=1$, we can obtain $B_{2}\left(x^{\prime}\right)$ such that for $m=1,2,3,4$,

$$
\operatorname{gcd}_{2}\left(x, S\left(x^{\prime}, v_{m}, z_{1}, z_{2}, z_{3}\right)\right)=1 \quad \text { for distinct } z_{i} \in B_{2}\left(x^{\prime}\right) .
$$

To do this, replace the proof of Lemma 3.5.1 by the following: Choose honest dual vectors $y$ to $\operatorname{pr}_{2}(x), y^{\prime}$ to $\operatorname{pr}_{2}\left(x^{\prime}\right)$ and $y_{j}$ to $\operatorname{pr}_{2}\left(v_{j}\right)$ by Proposition 2.2.9. $S=$ $\left\langle\operatorname{pr}_{2}(x), \operatorname{pr}_{2}\left(x^{\prime}\right)\right\rangle$ is a summand, its dual is $D=\left\langle y, y^{\prime}\right\rangle$, and we have $T$ such that $(S \oplus D) \oplus T=H_{2}$. Using the dual vectors, we can modify $\operatorname{pr}_{T}\left(v_{j}\right)$ to $v_{j}^{\prime}$, and $\operatorname{pr}_{2}(x), \operatorname{pr}_{2}\left(x^{\prime}\right)$ to $p(x), p\left(x^{\prime}\right)$, such that $p(x), p\left(x^{\prime}\right), v_{1}^{\prime}, \ldots, v_{4}^{\prime}$ is isotropic and $v_{1}^{\prime}, \ldots, v_{4}^{\prime}$ extends to a symplectic basis by $y_{1}, \ldots, y_{4}$. Finally replace $p(x)$ by $p(x)-v_{1}^{\prime}-v_{2}^{\prime}-v_{3}^{\prime}-v_{4}^{\prime}$. The result is extendable to a symplectic basis $B_{2}\left(x^{\prime}\right)$ of $\left\langle\operatorname{pr}_{2}(x), \operatorname{pr}_{2}\left(x^{\prime}\right)\right\rangle_{\mathbb{Q}}^{\perp}$ that satisfies the desired equation.

Lemma 3.5.3 for $i=2$ is more complicated:

Corollary 3.6.2 Let $g \geq 6$ and $n \leq 3$. Let $B_{2}$ be a basis of $\operatorname{pr}_{2}\left(\left\langle\operatorname{pr}_{2}(x)\right\rangle \frac{\perp}{\mathbb{Q}}\right)$ satisfying (41) in Remark 3.6.1. Assume $z_{1}, \ldots, z_{n} \in B_{2}$. Then:

(i) For $k \geq 2, \mathcal{B}_{Z, x, k}^{2}(g)$ is connected, and if $n \leq 2$, then the complex with $a_{1}$ removed, ie $\mathcal{B}_{Z, x, k}^{2}(g) \backslash\left(\operatorname{star}\left(a_{1}\right) \backslash \operatorname{link}\left(a_{1}\right)\right)$, is also connected.

(ii) For $k=1$, let $v_{1}, v_{2} \in \mathcal{B}_{Z, x, 1}^{2}(g)$, and assume that $\operatorname{gcd}_{2}\left(x, v_{1}, v_{2}\right)>0$ and $\operatorname{gcd}_{2}\left(x, S\left(v_{1}, v_{2}, z_{1}, z_{2}, z_{3}\right)\right)=1$. Then there exists a path $v_{1} \rightarrow w \rightarrow v_{2}$ in $\mathcal{B}_{Z, x, 1}^{2}(g)$ with $\operatorname{gcd}_{2}\left(x, v_{1}, v_{2}, w\right)>0$.

(iii) For $k=0$, the full subcomplex of $\mathcal{B}_{Z, x, 0}^{2}(g)$ spanned by the vertices $v$ with $\mathrm{rk}^{b_{1}}(x)=\mathrm{rk}^{b_{1}}(v)$ is connected. If $v_{1}, v_{2} \in \mathcal{B}_{Z, x, 0}^{2}(g)$ with $\mathrm{rk}^{b_{1}}(x)=\mathrm{rk}^{b_{1}}\left(v_{j}\right)$ and $\operatorname{gcd}_{2}\left(v_{j}, x\right)>0$, then there is a path $v_{1} \rightarrow w \rightarrow v_{2}$ in $\mathcal{B}_{Z, x, 0}^{2}(g)$ with $\operatorname{gcd}_{2}\left(x, v_{j}, w\right)>0$. 
Proof For $k \geq 2$, the first part is easy since $a_{1} \in \mathcal{B}_{Z, x, k}^{2}(g)$, and for any other $v \in \mathcal{B}_{Z, x, k}^{2}(g)^{(0)}$, we see $\left\{v, a_{1}\right\} \in \mathcal{B}_{Z, x, k}^{2}(g)$. For the second part of $k \geq 2$, let $v_{1}, v_{2} \in \mathcal{B}_{Z, x, k}^{2}(g)$ be given. Note $\operatorname{pr}_{2}(x)=x-a_{1}$ is simple. By identifying $H_{2} \cong$ $H(g)$, we can say $\operatorname{pr}_{2}\left(v_{j}\right)=v_{j}-a_{1} \in \mathcal{B}_{Z, \mathrm{pr}_{2} x, k-1}^{1}(g)$, since $z_{m} \in H_{2}$ already. Lemma 3.5.3 implies that $\mathcal{B}_{Z, \mathrm{pr}_{2} x, k-1}^{1}(g)$ is connected when $g \geq 6$, so there is a path $\operatorname{pr}_{2}\left(v_{1}\right)=w_{0}^{\prime} \rightarrow w_{1}^{\prime} \rightarrow \cdots \rightarrow w_{\ell}^{\prime}=\operatorname{pr}_{2}\left(v_{2}\right)$. Set $w_{j}=a_{1}+w_{j}^{\prime}$, then $v_{1}=w_{0} \rightarrow$ $w_{1} \rightarrow \cdots \rightarrow w_{\ell}=v_{2}$ is a path from $v_{1}$ to $v_{2}$ in $\mathcal{B}_{Z, x, k}^{2}(g)$.

For $k=0$, let $v_{1}, v_{2} \in \mathcal{B}_{Z, x, 0}^{2}(g)^{(0)}$ with $\operatorname{rk}^{b_{1}}\left(v_{j}\right)=\mathrm{rk}^{b_{1}}(x)$ be given. Since $v_{j} \in \mathcal{F}_{1}^{2}$, we get $\operatorname{pr}_{2}\left(v_{j}\right) \in \mathcal{B}_{Z, x, 1}^{1}(g+1)$. Since $g \geq 6, \mathcal{B}_{Z, x, 1}^{1}(g+1)$ is connected by Lemma 3.5.3, and from the proof we get a path $\operatorname{pr}_{2}\left(v_{1}\right) \rightarrow w^{\prime} \rightarrow \operatorname{pr}_{2}\left(v_{2}\right)$ in $\mathcal{B}_{Z, x, 1}^{1}(g+1)$. Then $w^{\prime} \in H_{2}$, and we set $w=a_{1}+\operatorname{rk}^{b_{1}}(x) b_{1}+w^{\prime} \in \mathcal{F}_{1}^{2}$. By the choice of $w^{\prime}$ in the proof, we see $v_{1} \rightarrow w \rightarrow v_{2}$ is a path in $\mathcal{B}_{Z, x, 0}^{2}(g)$, and $\operatorname{gcd}_{2}\left(x, w, v_{j}\right)=\operatorname{gcd}_{2}\left(x, v_{j}\right)$, which also shows the last part of $k=0$.

Finally, for $k=1$, we proceed similarly to the proof of Lemma 3.5.3 for $k=1$, except that we consider $V^{0}=\langle x\rangle, V^{1}=\left\langle v_{1}, v_{2}\right\rangle$, and $V^{2}=\left\langle z_{1}, z_{2}, z_{3}\right\rangle$. Projecting onto $H_{2}$, we take successive duals similar to (40), which becomes

$$
\left(S V_{2}^{0} \oplus D^{0}\right) \oplus\left(S V_{2}^{1,0} \oplus D^{1}\right) \oplus\left(S V_{2}^{2,1} \oplus D^{2}\right) \oplus T^{2}=H_{2} .
$$

Since $\operatorname{gcd}_{2}\left(x, S\left(v_{1}, v_{2}, z_{1}, z_{2}, z_{3}\right)\right)=1$, we have $S V_{2}^{0}=V_{2}^{0}=\left\langle\operatorname{pr}_{2}(x)\right\rangle$ and $D^{0}=\langle u\rangle$, where we can choose the dual $u \in H_{2}$ to be orthogonal to $v_{1}, v_{2}, z_{1}, z_{2}, z_{3}$. A dimension count shows that there is a simple vector $w^{\prime} \in S V_{2}^{2,1} \oplus D^{2}$ such that $w^{\prime} \perp z_{j}$ for $j=1,2,3$, and of course $w^{\prime} \perp x, v_{1}, v_{2}$. Set $w=a_{1}+c u+w^{\prime}$, where $c \in \mathbb{Z}$ is such that $w \perp x$. Then $w$ is orthogonal to $x, v_{1}, v_{2}, z_{1}, z_{2}, z_{3}$, and we see $v_{1} \rightarrow w \rightarrow v_{2}$ is a path in $\mathcal{B}_{Z, x, 1}^{2}(g)$. Moreover, by construction, $\operatorname{gcd}_{2}\left(x, v_{1}, v_{2}, w\right)=\operatorname{gcd}_{2}\left(x, v_{1}, v_{2}\right)>0$.

To prove Lemma 3.4.5 for $i=2$, we now need two steps. The first asserts the existence of the desired basis of $\bigwedge^{3}\left\langle x, x^{\prime}, b_{1}\right\rangle \frac{\perp}{\mathbb{Q}}$, under certain conditions:

Lemma 3.6.3 Let $x^{\prime} \in \mathcal{F}_{k+1}^{2}$ be given. Assume there are $x_{1}^{\prime}, x_{2}^{\prime}, x_{3}^{\prime}, x_{4}^{\prime} \in \mathcal{F}_{k+1}^{2}(g)^{(0)}$ such that $\left\{x, x_{j}^{\prime}\right\} \in \mathcal{F}_{k}^{2}$ for $j=1,2,3,4$, and there is a basis $\bar{B}\left(x_{j}^{\prime}\right)$ for $\bigwedge^{3}\left\langle x, x_{j}^{\prime}, b_{1}\right\rangle_{\mathbb{Q}}$ satisfying Lemma 3.4.5 for $j=1,2,3,4$. Assume further:

(a) If $k \geq 2$, that $x_{j}^{\prime}=A_{j}$ for $j \leq k-1$ and $\operatorname{gcd}_{2}\left(x, x^{\prime}, x_{2}^{\prime}, \ldots, x_{4}^{\prime}\right)>0$.

(b) If $k=1$, that $\operatorname{gcd}_{2}\left(x, x^{\prime}, x_{1}^{\prime}, \ldots, x_{4}^{\prime}\right)=1$.

(c) If $k=0$, that either

(1) $\left\{x, x^{\prime}, x_{1}^{\prime}, x_{2}^{\prime}, x_{3}^{\prime}, x_{4}^{\prime}\right\} \in \mathcal{B}^{2}(g)$, or

(2) $\operatorname{gcd}_{2}\left(x, x^{\prime}, x_{1}^{\prime}, \ldots, x_{4}^{\prime}\right)>0$ (if $\operatorname{pr}_{2}(x)=0$, omit $x$ ), and furthermore $\mathrm{rk}^{b_{1}}\left(x_{j}^{\prime}\right)=\mathrm{rk}^{b_{1}}\left(x^{\prime}\right)=\mathrm{rk}^{b_{1}}(x)$ for $j=1,2,3,4$.

Then there is a basis $\bar{B}\left(x^{\prime}\right)$ of $\bigwedge^{3}\left\langle x, x^{\prime}, b_{1}\right\rangle_{\mathbb{Q}}^{\perp}$ satisfying Lemma 3.4.5. 
Proof First, in case (c)(1), the result follows directly from Lemma 3.3.2. So consider the other cases. We claim that if $\operatorname{pr}_{2}(x) \neq 0$, then

$$
\left\langle x, x^{\prime}, b_{1}\right\rangle^{\perp}=\operatorname{pr}_{2}\left(\left\langle\operatorname{pr}_{2}(x), \operatorname{pr}_{2}\left(x^{\prime}\right)\right\rangle^{\perp}\right) \oplus\left\langle\tilde{b}_{1}\right\rangle,
$$

where $\tilde{b}_{1}=b_{1}-u$ for any $u \in H_{2}$ satisfying $i_{\text {alg }}(x, u)=i_{\text {alg }}\left(x^{\prime}, u\right)=1$. (43) follows since $\mathrm{rk}^{a_{1}}(x)=\mathrm{rk}^{a_{1}}\left(x^{\prime}\right)=1$ and by a dimension count.

We take a $\mathbb{Q}$-basis $B_{2}\left(x^{\prime}\right)$ of $\operatorname{pr}_{2}\left(\left\langle\operatorname{pr}_{2} x, \operatorname{pr}_{2} x^{\prime}\right\rangle^{\perp}\right)$, as in Remark 3.6.1 with $v_{j}=x_{j}^{\prime}$; note this is possible because of, respectively, (a), (b) or (c)(2). From it we get a $\mathbb{Q}$-basis $\bar{B}_{2}\left(x^{\prime}\right)$ for $\bigwedge^{3}\left(\operatorname{pr}_{2}\left(\left\langle\operatorname{pr}_{2} x, \operatorname{pr}_{2} x^{\prime}\right\rangle^{\perp}\right)\right)$.

Write $B_{2}\left(x^{\prime}\right)=\left\{z_{1}, \ldots, z_{n}\right\}$. Now suppose for each pair $z_{r}, z_{s} \in B_{2}\left(x^{\prime}\right)$ with $r<s$ we have chosen $u_{r s}$ satisfying $i_{\text {alg }}\left(x, u_{r s}\right)=i_{\text {alg }}\left(x^{\prime}, u_{r s}\right)=1$. Set $\tilde{b}_{1}(r, s)=b_{1}-u_{r s}$. Then by (43), the following defines a basis of $\bigwedge^{3}\left\langle x, x^{\prime}, b_{1}\right\rangle_{\mathbb{Q}}^{\perp}$ :

$$
\bar{B}\left(x^{\prime}\right)=\bar{B}_{2}\left(x^{\prime}\right) \cup\left\{\tilde{b}_{1}(r, s) \wedge z_{r} \wedge z_{s} \mid 1 \leq r<s \leq n\right\} .
$$

We will show that for each $z \in \bar{B}\left(x^{\prime}\right)$ there is an $N$ such that $\left(x^{\prime}, z\right) \in F_{N}$, and at the same time construct the $u_{r s}$.

First consider a given $z=z_{r} \wedge z_{s} \wedge z_{t} \in \bar{B}_{2}\left(x^{\prime}\right)$. Then there is $m \in\{1,2,3,4\}$ with $z \in$ $\bigwedge^{3}\left\langle x, x_{m}^{\prime}, b_{1}\right\rangle_{\mathbb{Q}}^{\perp}$. By assumption, $\left(z, x_{m}^{\prime}\right) \in F_{q}$ for some $q \in \mathbb{Z}$. The assumptions (a), (b) or (c)(2) ensures that Corollary 3.6.2 gives a path $x_{m}^{\prime}=w_{0} \rightarrow w_{1} \rightarrow \cdots \rightarrow w_{\ell}=x^{\prime}$ in $\mathcal{B}_{Z, x, k}^{2}(g)$ where $Z=\left\langle z_{r}, z_{s}, z_{t}\right\rangle^{\perp}$. (For $k=1$ use Remark 3.6.1.) Then Lemma 3.5.4 gives $\left(z, x^{\prime}\right) \in F_{q+\ell} .(*)$

This finishes the proof for $\operatorname{gcd}_{2}\left(x, x^{\prime}\right)=0$, since then $B\left(x^{\prime}\right)=B_{2}\left(x^{\prime}\right)$.

Now consider the other type of basis vector in $\bar{B}\left(x^{\prime}\right)$, so let $r<s$ be given. Take $m \in\{1,2,3,4\}$ with $z_{r} \wedge z_{s} \in \bigwedge^{2}\left\langle x, x_{m}^{\prime}, b_{1}\right\rangle \stackrel{\perp}{\mathbb{Q}}$.

For now, assume $k \neq 1$. By Corollary 3.6.2 with $Z=\left\langle z_{r}, z_{S}\right\rangle^{\perp}$, there is a path $x_{m}^{\prime}=v_{0} \rightarrow v_{1} \rightarrow \cdots \rightarrow v_{\ell}=x^{\prime}$ in $\mathcal{B}_{Z, x, k}^{2}(g)$ with $\operatorname{gcd}_{2}\left(x, v_{j}, v_{j-1}\right)>0$; for $k \geq 2$ this is because the path avoids $a_{1}$. Thus by Proposition 2.2.9, if we take $\mathbb{Q}$-coefficients, we can choose $u_{j} \in\left(H_{\mathbb{Q}}\right)_{2}$ such that

$$
i_{\text {alg }}\left(x, u_{j}\right)=i_{\text {alg }}\left(v_{j}, u_{j}\right)=i_{\text {alg }}\left(v_{j-1}, u_{j}\right)=1 .
$$

Now $\tilde{b}_{1}^{j}:=b_{1}-u_{j} \in\left\langle x, v_{j}, v_{j-1}\right\rangle^{\perp}$. For $j=0$ just use $v_{0}=x_{m}^{\prime}$ to get $\tilde{b}_{1}^{0} \in\left\langle x, x_{m}^{\prime}\right\rangle^{\perp}$. We set $u_{r, s}=u_{\ell}$.

Write $z^{j}=\widetilde{b}_{1}^{j} \wedge z_{r} \wedge z_{s}$. Inductively assume there is $n(j) \in \mathbb{N}$ such that $\left(z^{j}, w_{j}\right) \in F_{n(j)}$. The induction start is the assumption on $\bar{B}\left(x_{m}^{\prime}\right)$. For the induction step, suppose $\left(z^{j}, v_{j}\right) \in F_{n(j)}$. Consider the difference $z^{j+1}-z^{j}=\left(u_{j+1}-u_{j}\right) \wedge z_{2} \wedge z_{3}$. Since 
$\left(u_{j+1}-u_{j}\right), z_{r}, z_{s} \in \operatorname{pr}_{2}\left(\left\langle\operatorname{pr}_{2}(x), \operatorname{pr}_{2}\left(v_{j}\right)\right\rangle_{\mathbb{Q}}^{\perp}\right)$, we can apply $(*)$ above for $x^{\prime}=v_{j}$ and get $p(j) \in \mathbb{N}$ with $\left(z^{j+1}-z^{j}, v_{j}\right) \in F_{p(j)}$. Combined with the induction hypothesis, we see $\left(z^{j+1}, v_{j}\right) \in F_{\max }(p(j), n(j))$. Since $\left\{x, v_{j}, v_{j+1}\right\}$ is a simplex, $\left(z^{j+1}, v_{j+1}\right) \in F_{n(j+1)}$ where $n(j+1)=\max (p(j), n(j))+1$. This finishes the induction. We have shown that $z=\widetilde{b}_{1}(r, s) \wedge z_{r} \wedge z_{s}$ satisfies $\left(z, x^{\prime}\right) \in F_{n(\ell)}$,

For $k=1$, the path $x_{m}^{\prime} \rightarrow w \rightarrow x^{\prime}$ that is provided by Corollary 3.6.2 satisfies $\operatorname{gcd}_{2}\left(x, x_{m}^{\prime}, w, x^{\prime}\right)>0$, so there is now $u \in\left(H_{\mathbb{Q}}\right)_{2}$ with

$$
i_{\text {alg }}(x, u)=i_{\text {alg }}\left(x_{m}^{\prime}, u\right)=i_{\text {alg }}(w, u)=i_{\text {alg }}\left(x^{\prime}, u\right)=1 .
$$

Then choose $u_{r, s}=u$. Write $z=\left(b_{1}-u\right) \wedge z_{r} \wedge z_{s}$. Lemma 3.5.4 gives that $\left(z, x^{\prime}\right) \in F_{N}$ for some $N$, because $\bar{B}\left(x_{m}^{\prime}\right)$ satisfies Lemma 3.4.5.

With this, we can show Lemma 3.4.5 for $i=2$ :

Proof of Lemma 3.4.5 for $i=2$ First, if $k \geq 2$ : We have $x_{1}, \ldots, x_{5} \in \mathcal{F}_{k+1}^{2}$ from Lemma 3.3.7, and we use as $x_{1}^{\prime}, \ldots, x_{4}^{\prime}$ four among $x_{1}, \ldots, x_{5}$ such that Lemma 3.6.3(a) holds. From Remark 3.4.6 there are bases $\bar{B}\left(x_{m}\right)$ as in Lemma 3.4.5. Now Lemma 3.6.3 gives the basis $\bar{B}\left(x^{\prime}\right)$ as desired.

If $k=1$ we consider two cases: First, if $\operatorname{gcd}_{2}\left(x, x^{\prime}, x_{1}, x_{2}, x_{3}, x_{4}\right)=1$, then we argue as in the case $k \geq 2$ with $x_{j}^{\prime}=x_{j}$ for $j=1,2,3,4$, and are done.

If not, then we will find $x_{1}^{\prime}, \ldots, x_{4}^{\prime} \in \mathcal{F}_{2}^{2}$ such that

$$
\operatorname{gcd}_{2}\left(x, x_{1}^{\prime}, x_{2}^{\prime}, x_{3}^{\prime}, x_{4}^{\prime}, x_{1}, x_{2}, x_{3}, x_{4}\right)=\operatorname{gcd}_{2}\left(x, x^{\prime}, x_{1}^{\prime}, x_{2}^{\prime}, x_{3}^{\prime}, x_{4}^{\prime}\right)=1 .
$$

Then Lemma 3.6.3 gives the basis $\bar{B}\left(x_{j}^{\prime}\right)$ for each $j=1,2,3,4$ by using $x_{1}, x_{2}, x_{3}, x_{4}$, which by Lemma 3.6.3 along with (45) gives the basis $\bar{B}\left(x^{\prime}\right)$.

To construct these $x_{j}^{\prime}$, let $S=S\left(x, x^{\prime}, x_{1}, \ldots, x_{4}, y\right) \subseteq H_{2}$, where $y \in H_{2}$ denotes a dual vector to $\operatorname{pr}_{2}(x)$, which is simple since $k=1$. We can employ $\operatorname{pr}_{2}(x), \operatorname{pr}_{2}\left(x_{1}\right)$, $\ldots, \operatorname{pr}_{2}\left(x_{4}\right)$ as part of a $\mathbb{Z}$-basis of $S$, since $\left\{x, x_{1}, x_{2}, x_{3}, x_{4}\right\} \in \mathcal{F}_{1}^{2}$. Obviously, $\operatorname{dim} S \leq 7$, and since $\operatorname{dim} H_{2}=2 g \geq 12$, we can extend this basis of $S$ by four basis vectors, call them $v_{1}, v_{2}, v_{3}, v_{4}$. Set $x_{j}^{\prime}=a_{1}+v_{j}+c_{j} y$ for $j=1,2,3,4$. Here $c_{j} \in \mathbb{Z}$ is chosen such that $x_{j}^{\prime} \perp x$, ie $c_{j}=-i_{\text {alg }}\left(x, a_{1}+v_{j}\right)$. Now (45) follows from the fact that

$$
1=\operatorname{gcd}_{2}\left(S, v_{1}, v_{2}, v_{3}, v_{4}\right)=\operatorname{gcd}_{2}\left(S, x_{1}^{\prime}, x_{2}^{\prime}, x_{3}^{\prime}, x_{4}^{\prime}\right) .
$$

Last, if $k=0$, we again consider two cases. First, if $\mathrm{rk}^{b_{1}}\left(x^{\prime}\right)=\mathrm{rk}^{b_{1}}(x)$. From Lemma 3.3.7(iv) we have $\mathrm{rk}^{b_{1}}\left(x_{j}\right)=\mathrm{rk}^{b_{1}}(x)$ for $j=1,2,3,4,5$, so we can do as in $k \geq 2$. 
If not, we get from Lemma 3.3.7 that $\left\{x, x^{\prime}\right\}^{*}=\left\{x_{1}^{\prime}, \ldots, x_{4}^{\prime}\right\}$ satisfies

$$
\left\{x, x^{\prime}, x_{1}^{\prime}, \ldots, x_{4}^{\prime}\right\} \in \mathcal{F}_{0}^{2} \quad \text { and } \quad \mathrm{rk}^{b_{1}}\left(x_{j}^{\prime}\right)=\mathrm{rk}^{b_{1}}(x) .
$$

Then by the first part of $k=0$, we have a basis $\bar{B}\left(x_{j}^{\prime}\right)$ for $j=1,2,3,4$, and thus by Lemma 3.6.3 we also get $\bar{B}\left(x^{\prime}\right)$ satisfying Lemma 3.4.5.

\section{References}

[1] A Borel, Stable real cohomology of arithmetic groups II, from: "Manifolds and Lie groups (Notre Dame, IN, 1980)", (S Murakami, J Hano, K Okamoto, A Morimoto, H Ozeki, editors), Progr. Math. 14, Birkhäuser, Mass. (1981) 21-55 MR642850

[2] K S Brown, Cohomology of groups, Graduate Texts in Math. 87, Springer, New York (1994) MR1324339

[3] T Church, B Farb, Representation theory and homological stability arXiv: 1008.1368v1

[4] J L Harer, Stability of the homology of the mapping class groups of orientable surfaces, Ann. of Math. 121 (1985) 215-249 MR786348

[5] N V Ivanov, Complexes of curves and Teichmüller modular groups, Uspekhi Mat. Nauk 42 (1987) 49-91, 255 MR896878 In Russian; translated in Russian Math. Surveys 42 No 3 (1987) 55-107

[6] A Putman, The Johnson homomorphism and its kernel arXiv:0904.0467v2

[7] A Putman, Cutting and pasting in the Torelli group, Geom. Topol. 11 (2007) 829-865 MR2302503

[8] A Putman, An infinite presentation of the Torelli group, Geom. Funct. Anal. 19 (2009) 591-643 MR2545251

[9] O Randal-Williams, Resolutions of moduli spaces and homology stability arXiv: 0909.4278v1

[10] B van den Berg, On the abelianization of the Torelli group, $\mathrm{PhD}$ thesis, Universiteit Utrecht (2003) Available at http://igitur-archive.library.uu.nl/ dissertations/2003-1013-162512/inhoud.htm

Mathematical Institute, University of Bonn

Endenicher Allee 60, D-53115 Bonn, Germany

Department of Mathematics, Aarhus University

Klokkerfaldet 109, DK-8210 Aarhus, Denmark

soren_boldsen@lycos.com, miahauge@hotmail.com

Proposed: Benson Farb

Seconded: Danny Calegari, Jesper Grodal

Received: 4 September 2011 Accepted: 14 March 2012 